\title{
Architecture and evolution of the cis-regulatory system of the echinoderm kirrelL gene
}

14 


\section{Abstract}

The gene regulatory network (GRN) that underlies echinoderm skeletogenesis is a prominent model of GRN architecture and evolution. Kirrel $L$ is an essential downstream effector gene in this network and encodes an Ig-superfamily protein required for the fusion of skeletogenic cells and the formation of the skeleton. In this study, we dissected the transcriptional control region of the kirrell gene of the purple sea urchin, Strongylocentrotus purpuratus. Using plasmid- and BAC-based transgenic reporter assays, we identified key cis-regulatory elements (CREs) and transcription factor inputs that regulate $S p$-kirrelL, including direct, positive inputs from two key transcription factors in the skeletogenic GRN, Alx1 and Ets1. We next identified kirrelL cis-regulatory regions from seven other echinoderm species that together represent all classes within the phylum. By introducing these heterologous regulatory regions into developing sea urchin embryos we provide evidence of their remarkable conservation across $\sim 500$ million years of evolution. We dissected in detail the kirrelL regulatory region of the sea star, Patiria miniata, and demonstrated that it also receives direct inputs from Alx1 and Ets1. Our findings identify kirrelL as a component of the ancestral echinoderm skeletogenic GRN. They support the view that GRN sub-circuits, including specific transcription factor-CRE interactions, can remain stable over vast periods of evolutionary history. Lastly, our analysis of kirrelL establishes direct linkages between a developmental GRN and an effector gene that controls a key morphogenetic cell behavior, cell-cell fusion, providing a paradigm for extending the explanatory power of GRNs. 


\section{Introduction}

Evolutionary changes in animal form have occurred through modifications to the developmental programs that give rise to anatomy. These developmental programs can be viewed as gene regulatory networks (GRNs, complex, dynamic networks of interacting regulatory (i.e., transcription factor-encoding) genes that determine the transcriptional states of embryonic cells (Peter and Davidson, 2015). Sea urchins and other echinoderms are prominent models for GRN biology for several reasons: (1) there are well-developed tools for dissecting developmental GRNs in these animals, (2) a large number of species that represent a wide range of evolutionary distances are amenable to study, and (3) there is a rich diversity of developmental modes and morphologies within the phylum (Arnone et al., 2016).

All adult echinoderms possess elaborate, calcified endoskeletons. Most species are maximal indirect developers; i.e., they develop via a feeding larva that undergoes metamorphosis to produce the adult. The feeding larvae of echinoids (sea urchins) and ophiuroids (brittle stars) have extensive endoskeletons, holothuroids (sea cucumbers) have rudimentary skeletal elements, and asteroids (sea stars) lack larval skeletal elements entirely. Larval skeletons are thought to be derived within the echinoderms as the feeding larvae of hemichordates (acorn worms), the sister group to echinoderms, and the larvae of crinoids (sea lilies and feather stars), a basal echinoderm clade, lack skeletons. The skeletal cells of larval and adult echinoderms are similar in many respects, supporting the widely accepted view that the larval skeleton arose via co-option of the adult skeletogenic program (Czarkwiani et al., 2013; Gao et al., 2015; Gao and Davidson, 2008; Killian et al., 2010; Mann et al., 2010, 2008; Richardson et al., 1989).

The embryonic skeleton of euechinoid sea urchins, the best studied taxon, is formed by a specialized population of skeletogenic cells known as primary mesenchyme cells (PMCs). These cells are the progeny of the large micromeres (LMs), four cells that arise near the vegetal pole during early cleavage. The GRN that underlies PMC specification is one of the best characterized GRNs in any animal embryo (Oliveri et al., 2008; Shashikant et al., 2018a). This GRN is initially deployed through the activity of a localized maternal protein, Dishevelled, which stabilizes B-catenin in the LM lineage, leading to the early zygotic expression of a repressor, pmar1/micro1 (Logan et al., 1999; Nishimura et al., 2004; Oliveri et al., 2002; Peng and Wikramanayake, 2013; Weitzel et al., 2004). These molecular events lead to the zygotic expression of several regulatory genes selectively in the LM-PMC lineage. Two of the most important of these regulatory genes are alx1 (Ettensohn et al., 2003) and ets 1 (Kurokawa et al., 1999), each of which is required for $\mathrm{PMC}$ specification and morphogenesis.

After their specification, PMCs undergo a spectacular sequence of morphogenetic behaviors that includes epithelial-mesenchymal transition (EMT), directional cell 
migration, cell fusion, and biomineral formation. PMCs undergo EMT at the late blastula stage, ingressing from the vegetal plate into the blastocoel. They migrate along the blastocoel wall and gradually arrange themselves in a ring-like pattern near the equator of the embryo. As they migrate, PMCs extend filopodia that fuse with those of neighboring PMCs, giving rise to a cable-like structure that joins the cells in a single, extensive syncytium. Beginning late in gastrulation and continuing throughout the remainder of embryogenesis, PMCs deposit calcified biomineral within the syncytial filopodial cable.

The complex sequence of PMC morphogenetic behaviors is regulated by hundreds of specialized effector proteins. The spatio-temporal expression patterns of these proteins are controlled by the GRN deployed in the LM-PMC lineage. A major current goal is to identify effector proteins that regulate specific PMC behaviors and elucidate the GRN circuitry that controls these genes (see Ettensohn, 2013; Lyons et al., 2012). Dissection of the cis-regulatory elements (CREs) that control essential morphogenetic effector genes, including the identification of specific transcription factor inputs, would directly link them to the relevant circuitry and provide a GRN-level explanation of developmental anatomy. At present, we have only a limited understanding of the cis-regulatory control of three PMC effector genes: two genes (sm30 and sm50) that encode secreted proteins occluded in the biomineral (Makabe et al., 1995; Walters et al., 2008) and a third gene (cyclophilin/cyp1) of unknown function (Amore and Davidson, 2006).

KirrelL is a PMC-specific, Ig domain-containing, transmembrane protein required for cell-cell fusion (Ettensohn and Dey, 2017). In kirrelL morphants, PMCs extend filopodia and migrate but filopodial contacts do not result in fusion; this prevents the formation of the PMC syncytium and results in the secretion of small, unconnected biomineralized elements. In all echinoderms that have been examined, the kirrell gene lacks introns, raising the possibility that its origin early in echinoderm evolution was a consequence of retrotransposition, a common gene transfer mechanism that results in intronless genes and one that has played a particularly prominent role in the diversification of Ig-domain-containing proteins (Baertsch et al., 2008; Cordaux and Batzer, 2009; Dermody et al., 2009; Farré et al., 2017). In sea urchins, kirrelL is expressed in a temporal and spatial pattern characteristic of many PMC effector genes. The gene is first expressed at the blastula stage ( 18 hpf) and peaks in expression early in gastrulation ( 30 hpf) (Tu et al., 2014). Expression then declines and is followed by a second peak at $\sim 64 \mathrm{hpf}$, when kirrelL is expressed predominantly at sites of active skeletal rod growth as a consequence of localized, ectoderm-derived cues (Sun and Ettensohn, 2014). Sp-kirrelL, like many PMC effector genes, is positively regulated both by Alx1 and Ets1 (Rafiq et al., 2014). Although the gene has only been studied in detail in sea urchins, a recent study found that kirrelL is also expressed specifically in the embryonic skeletogenic mesenchyme of a brittle star, Amphiura filiformis (Dylus et al., 2018). 
In the present study, we used plasmid- and BAC-based transgenic reporter assays to identify key cis-regulatory elements (CREs) and transcription factor inputs that regulate

128 kirrelL in the sea urchin, Strongylocentrotus purpuratus, directly linking this

129 morphogenetic effector gene to the PMC GRN. In addition, we identified kirrelL cis-

130 regulatory regions in echinoderm species from all major clades within the phylum and

131 found that these regulatory regions drove PMC-specific expression in developing sea

132 urchin embryos, highlighting their striking conservation across 450-500 million years of

133 evolution. We analyzed in detail the kirrelL regulatory region of the sea star, Patiria

134 miniata, and found that this gene, like Sp-kirrelL, receives direct inputs from Alx1 and

135 Ets1. Our findings identify kirrelL as a component of the ancestral echinoderm 136 skeletogenic GRN and strengthen the view that GRN sub-circuits, including specific 137 transcription factor-CRE interactions, can remain stable over very long periods of 138 evolutionary history. 


\section{Results}

140 The sea urchin Sp-kirrell cis-regulatory landscape

We identified potential Sp-kirrell CREs based on several criteria. We considered whether candidate regions were (1) hyperaccessible in PMCs relative to other cell types, (2) bound by Alx1, a key transcription factor in the PMC GRN and a positive regulator of Sp-kirrelL, (3) associated with active enhnacer RNA (eRNA) expression, and (4) evolutionary conserved. In a previous study, ATAC-seq and DNase-seq were used to identify regions of chromatin that are differentially accessible in PMCs relative to other cell types at the mesenchyme blastula stage (Shashikant et al., 2018b). ChIP-seq was used to indentify binding sites of Sp-Alx1 at the same developmental stage (Khor et al., 2019). Recently, we used Cap Analysis of Gene Expression Sequencing (CAGE-seq) to profile enhancer RNA (eRNA) expression at nine different stages of early sea urchin embryogenesis (Khor et al., 2021). Significantly, our integration of these different genome-wide datasets revealed several putative CREs located near Sp-kirrelL, some of which were found to share several signatures (Figure 1A). Developmental CAGE-seq profiles of eRNAs also provided additional information regarding temporal patterns of CRE activity (Figure 1B). To assist in identifying candidate CREs regulating the spatiotemporal expression of Sp-kirrelL, we used GenePalette (Smith et al., 2017) to perform phylogenetic footprinting of the $S$. purpuratus and $L$. variegatus kirrelL gene loci. Based on cross-species sequence conservation, cell type-specific DNA accessibility, Sp-Alx1 binding, and eRNA expression, we divided the intergenic sequences flanking Sp-kirrelL into 9 putative CREs (labeled elements A-K) (Figure 1C). The elements were between 1.0 to $2.4 \mathrm{~kb}$ in size, with an average size of $1.5 \mathrm{~kb}$. 
bioRxiv preprint doi: https://doi.org/10.1101/2021.08.27.457814; this version posted August 28, 2021. The copyright holder for this preprint (which was not certified by peer review) is the author/funder, who has granted bioRxiv a license to display the preprint in perpetuity. It is made available under aCC-BY 4.0 International license.

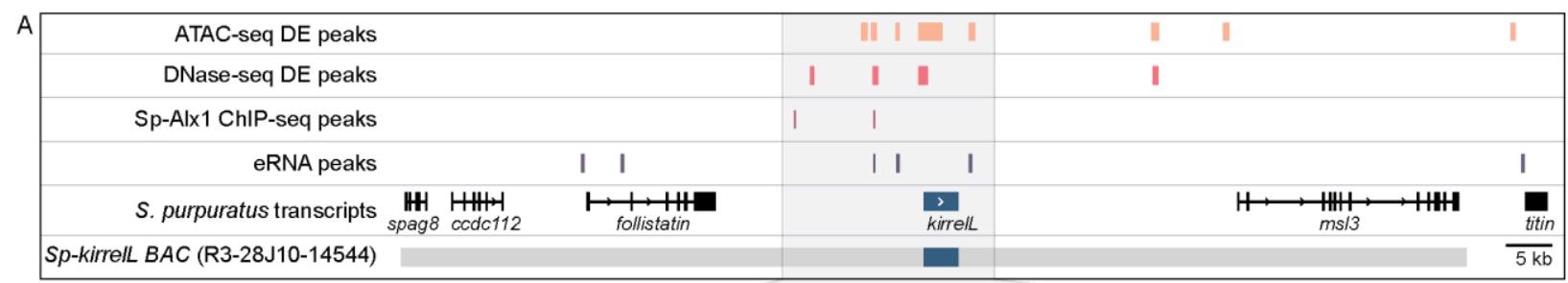
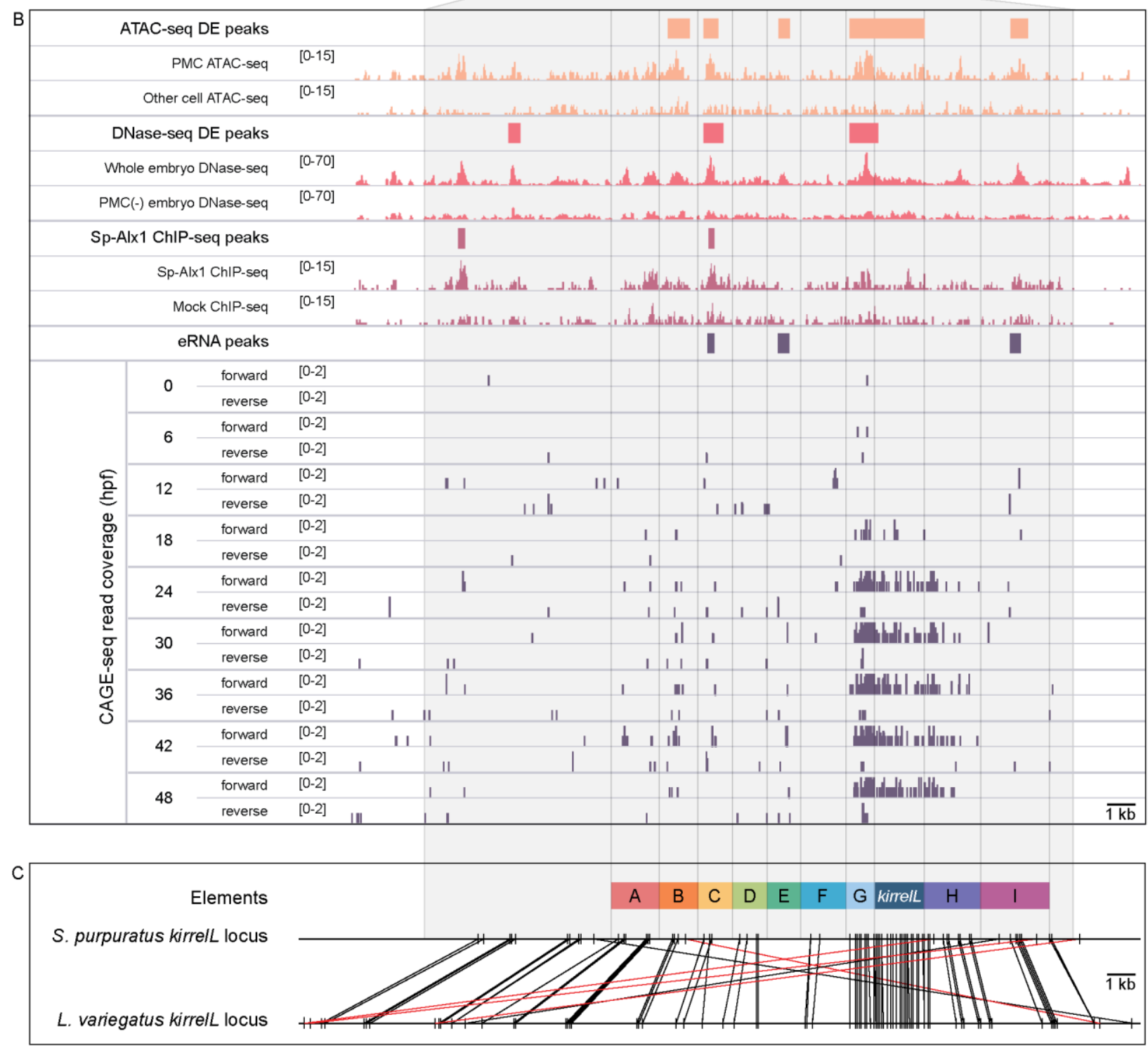

Figure 1: Characterization of the transcriptional regulatory landscape surrounding the $S$. purpuratus kirrelL (Sp-kirrelL) locus. (A) Diagram of the Sp-kirrelL locus showing neighboring genes, regions of chromatin differentially accessible in primary mesenchyme cells (PMCs) (ATAC-seq DE peaks and DNase-seq DE peaks) (Shashikant et al., 2018), Sp-Alx1 binding sites (Sp-Alx1 ChIP-seq peaks) (Khor et al., 2019), and eRNA peaks (Khor et al., 2021). (B) Signal obtained from each assay in the vicinity of the Sp-kirrelL locus. (C) Phylogenetic footprinting of genomic sequences near $S$. purpuratus and $L$. variegatus kirrelL ( $\pm 10 \mathrm{~kb}$ of an exon) using 
GenePalette. Black lines indicate identical sequences of $15 \mathrm{bp}$ or longer in the same orientation while red lines indicate identical sequences of $15 \mathrm{bp}$ or longer in the opposite orientation. 9 putative CREs (labeled elements A-I) were identified based on sequence conservation and chromatin signatures.

\section{Characterization of functional Sp-kirrelL cis-regulatory elements}

To test the transcriptional activity of the candidate cis-regulatory elements (Figure 2A), we cloned them individually or in combination into the EpGFPII reporter plasmid, which contains a weak, basal sea urchin promoter, derived from the $S p$-endo16 gene, upstream of GFP (see Materials and Methods) and injected them into fertilized eggs. We observed that a GFP reporter construct containing upstream elements $A$ to $G$ recapitulated the correct spatial expression pattern of endogenous $S p$-kirrelL with minimal ectopic expression (Figure 2B,C and Supplemental Table S1). Further dissections revealed that a reporter construct containing elements $D, E, F$, and $G$ also drove strong GFP expression specifically in PMCs while a construct consisting of elements $A, B$, and $C$ showed weak GFP expression in PMCs. When elements were tested individually, we found that only elements $\mathrm{C}$ and $\mathrm{G}$ were able to drive GFP expression in sea urchin embryos. Element $\mathrm{G}$, which is directly upstream of the $S p$-kirrelL translational start site and contains part of the Sp-kirrelL 5' untranslated region (UTR), was observed to drive strong GFP expression specifically in the PMCs. Element $C$ was also observed to drive GFP expression specifically in the PMCs, albeit much at lower levels than element G. 

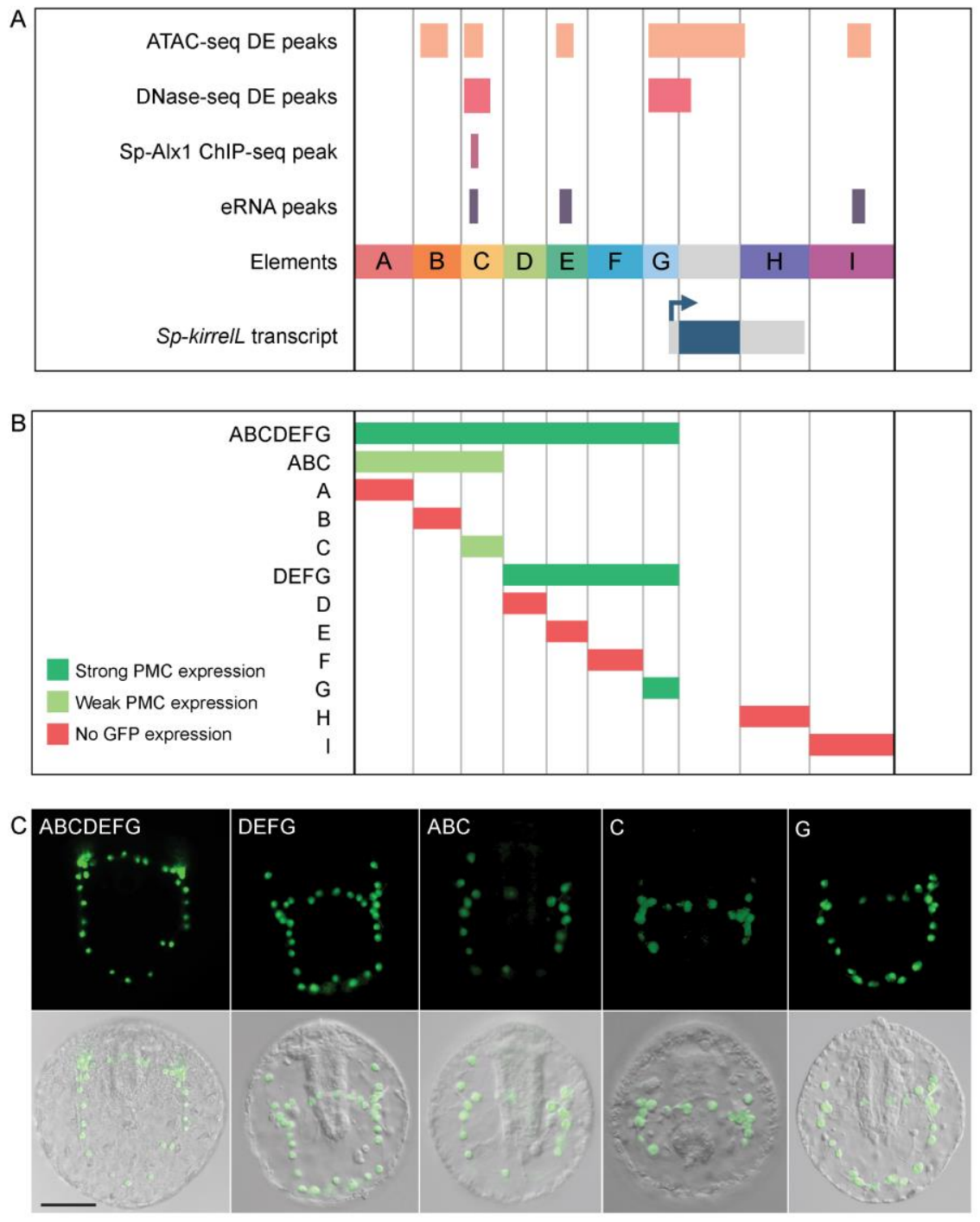

192 Figure 2: Functional analysis of non-coding genomic sequences flanking Sp-kirrelL to identify cisregulatory elements (CREs). (A) 9 putative CREs (labeled elements A-K) were identified based on sequence conservation and previously published datasets (Khor et al., 2021, 2019; Shashikant et al., 2018b). (B) Summary of GFP expression regulated by putative CREs, as assessed by transgenic reporter assays. Strong PMC expression is defined as $>15 \%$ of total injected embryos expressing GFP, with most exhibiting expression in PMCs only. Weak PMC expression is defined as $<15 \%$ of total injected embryos expressing GFP, with most exhibiting expression in PMCs only. (C) Spatial expression patterns of GFP reporter constructs containing different Sp-kirrelL elements at 48 hours post fertilization (hpf). Top row: GFP fluorescence. Bottom row: GFP 
Identification of direct transcriptional inputs into element C

We next focused on the molecular dissection of element $C$ to identify direct transcriptional inputs into this CRE. Element $C$ is noteworthy as it is differentially accessible in the PMCs based on both ATAC-seq and DNase-seq, bound by Sp-Alx1, and associated with eRNA expression (Figure 3A). We first performed a detailed dissection of element $C$ to identify the minimal region that supported strong, PMC-specific GFP expression. We found that a reporter construct containing element $C$ alone showed relatively weak reporter activity, similar to the construct containing elements $A, B$, and $C$ (Figure S1A). In contrast, a larger, overlapping CRE we termed BC.ATAC, which included part, but not all, of element $C$, exhibited strikingly enhanced GFP expression in PMCs. This difference in activity between element $C$ and BC.ATAC suggested that element $\mathrm{C}$ might contain regulatory sites that have greater activity when in close proximity to the promoter.

To explore this further, we generated several reporter constructs consisting of truncated forms of element $\mathrm{C}$, with boundaries defined by peaks from ATAC-seq (C.ATAC), DNase-seq (C.DNase), and Sp-Alx1 ChIP-seq (C.ChIP). The minimal element $C$ region that showed strong, PMC-specific activity was determined to be C.ChIP. Increasing the distance between the C.ChIP element and the promoter (as in the C.DNase construct) significantly reduced enhancer activity. To predict transcription factor inputs within C.ChIP, we scanned the $200 \mathrm{bp}$ C.ChIP sequence using JASPAR (Mathelier et al., 2016), with a focus on transcription factors known to be differentially expressed in the PMCs. This analysis identified several candidate Alx1 and Ets 1 binding sites (Figure 3B and Figure S1B,C). Consistent with previous RNA-seq analysis which has shown that Sp-kirrelL is sensitive to al $x 1$ and ets 1 knockdowns (Rafiq et al., 2014), our mutational analysis of C.ChIP revealed that mutations of all putative Alx1 and/or Ets1 binding sites completely abolished GFP expression (Figure 3C and Figure S1D). In contrast, constructs containing mutations in putative Fox or Meis binding sites exhibited reporter activity similar to that of the parental construct. Mutations of individual Alx1 and Ets1 sites revealed that Alx 1 half site 2 and Ets 1 site 1 provided key regulatory inputs. 

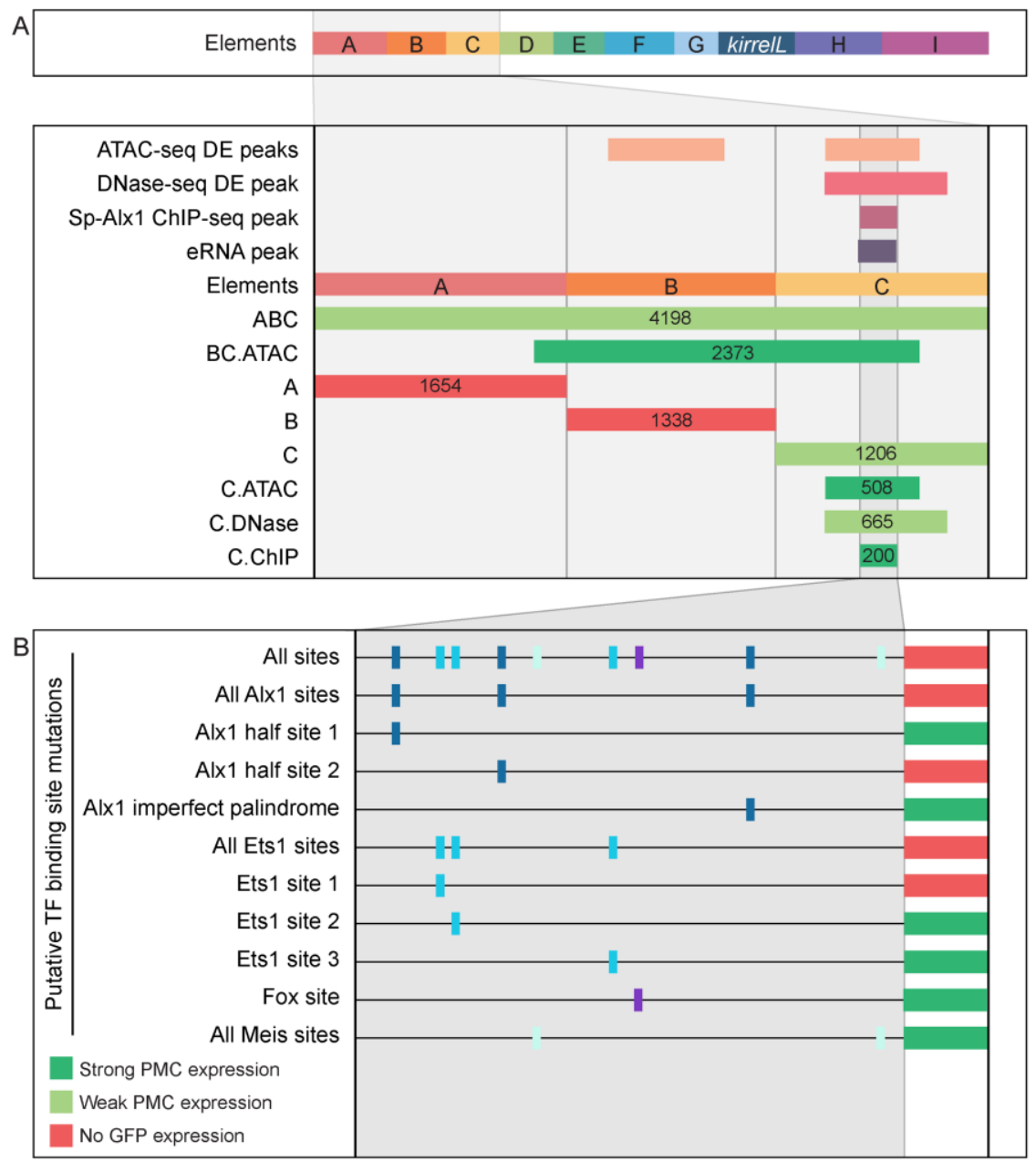

232 Figure 3: Molecular dissection of element $\mathrm{C}$ and the identification of direct transcriptional inputs.

233 (A) Summary of GFP expression regulated by element $\mathrm{C}$ truncations using reporter constructs.

234 Serial truncation of element $\mathrm{C}$ was performed based on boundaries of peaks defined by chromatin 235 accessibility, Sp-Alx1 binding, and eRNA expression. (B) Summary of GFP expression driven by 236 C.ChIP element mutants. Criteria for strong and weak PMC expression are defined in Figure 2. 

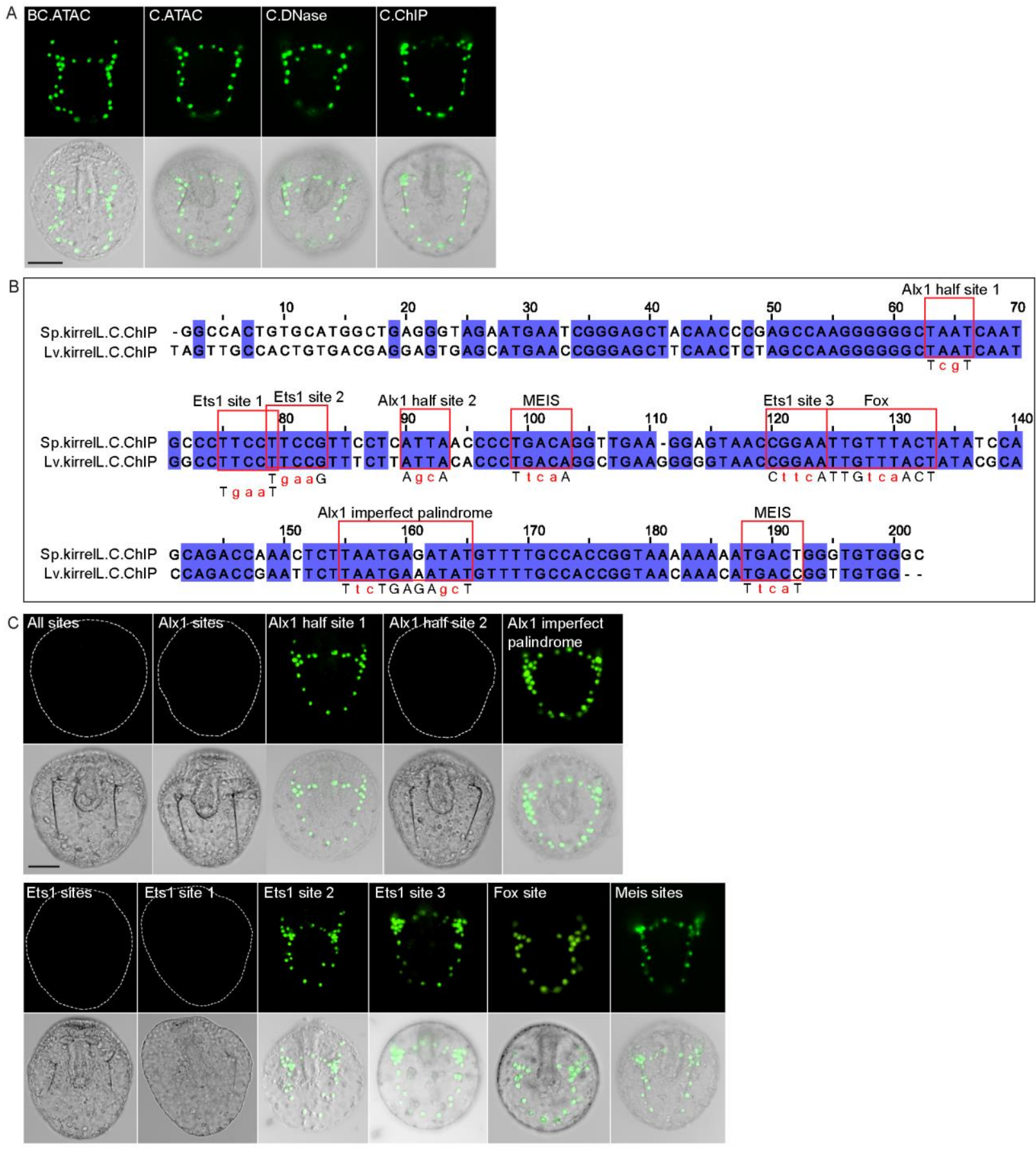

238 Figure S1: Element $\mathrm{C}$ truncation and mutational analysis. (A) Spatial expression patterns of GFP 239 reporter constructs containing different element $\mathrm{C}$ truncations at $48 \mathrm{hpf}$. (B) Cluster Omega 240 alignment of Sp-kirrelL and Lv-kirrelL C.ChIP sequences. Violet shading indicates conserved 241 sequences. Red boxes highlight putative transcription factor binding sites. (C) Spatial expression 242 patterns of GFP reporter constructs containing different C.ChIP element mutants at 48 hpf. Dotted 243 lines show outline of embryos that did not show GFP expression. Top rows: GFP fluorescence. 244 Bottom rows: GFP fluorescence overlayed onto DIC images. Scale bar: $50 \mu \mathrm{m}$. 
Analysis of the Sp-kirrelL promoter (Element G)

To characterize the native $S p$-kirrel $L$ promoter, we performed a detailed dissection of element $\mathrm{G}$, which contains sequences directly upstream of the $S p$-kirrelL translational start site, including the region encoding the Sp-kirrelL the 5' UTR (Figure 4A and Figure S2A). We found that a $301 \mathrm{bp}$ region surrounding the transcriptional start site, a region we considered to be the Sp-kirrelL promoter, was able to drive GFP expression in sea urchin embryos. The majority of expressing embryos, however, exhibited ectopic GFP expression, suggesting that $\mathrm{Sp}$-kirrelL core promoter is a strong, ubiquitous promoter. We next performed mutational analysis of the minimal element $G$ fragment that drove strongest PMC-specific GFP expression (G.ATAC). We determined that this CRE receives direct and positive inputs from Alx1 and Ets1, similar to the C.ChIP element (Figure 4B and Figure S3A,B). Reporter constructs with mutated Cebpa, Fos::Jun, Fox, Meis, and Tbrain binding sites exhibited similar PMC-specific GFP expression similar to that of the parental construct. We also injected the different Sp-kirrelL element $G$ truncations into fertilized $L$. variegatus eggs and observed similar expression patterns, indicating that inputs into element $G$ are conserved in these two sea urchin species (Figure S2B).

Our analysis of the native Sp-kirrelL promoter prompted us to investigate whether the addition of this region to our EpGFPII reporter constructs would allow us to uncover interactions between CREs and the native promoter that would have otherwise been missed (Figure S4A). Strikingly, we found that elements B, C, E, F, H, and I were individually able drive strong PMC-specific GFP expression when cloned adjacent to the $S p$-kirrelL promoter, although these elements had previously exhibited minimal activity in the context of the Sp-endo16 promoter alone (Figure 4C and Figure S4B; compared to Figure 2). We also observed that the presence of the native Sp-kirrelL promoter mitigated the need for the C.ChIP element within element $C$ to be adjacent to the promoter for strong PMC-specific GFP expression. We confirmed that enhancer activity was dependent on the sequence of the Sp-kirrelL promoter, as GFP expression was abolished in a construct where the sequence was shuffled (Figure S4C,D). Taken together, these findings showed that several CREs are capable of interacting specifically with the native $S p$-kirrelL promoter and this interaction can bypass spacing hurdles that are evident when the $\mathrm{Sp}$-endo16 promoter alone is present. 

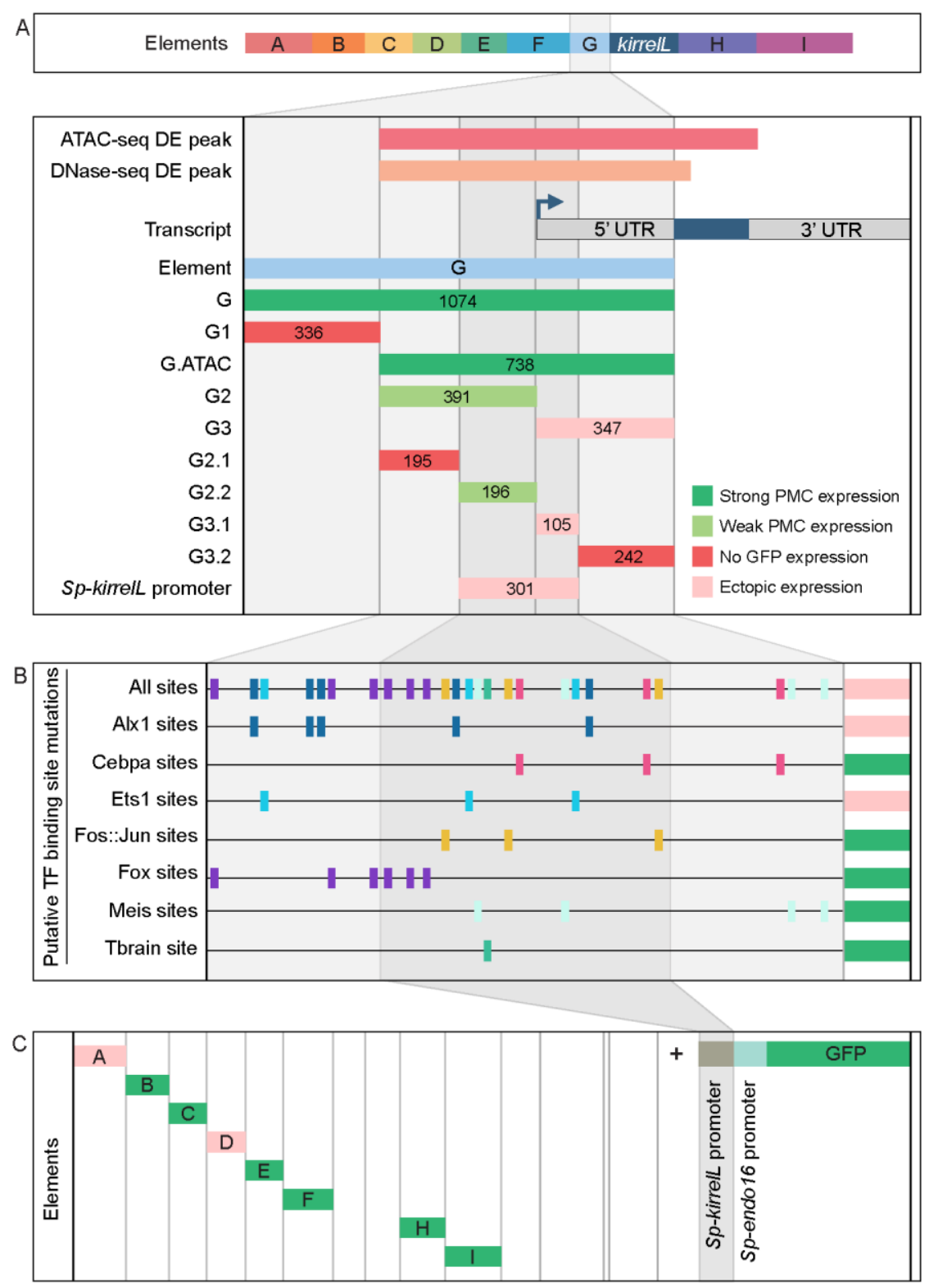

278 Figure 4: Molecular dissection and mutation of element G. (A) Summary of GFP expression 279 regulated by element $G$ truncations using EpGFPII reporter constructs. Serial truncation of 280 element $G$ was performed based on boundaries defined by chromatin accessibility and the kirrelL 5' UTR. Criteria for strong and weak PMC expression are defined in Figure 2. Ectopic expression is defined as $>10 \%$ of total injected embryos expressing GFP, with a majority exhibiting expression in cells other than PMCs. (B) Summary of GFP expression driven by G.ATAC element mutants using EpGFP// reporter constructs. (C) Analysis of element enhancer activity in modified EpGFPII reporter constructs containing the endogenous $S p$-kirrelL promoter elements. 


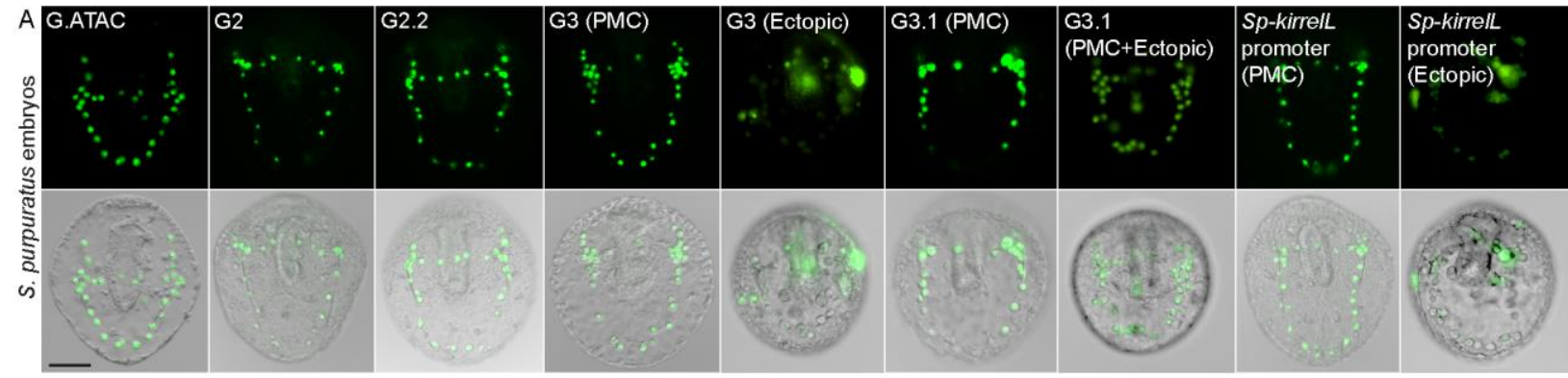

287 Figure S2: Element G truncation and mutational analysis. (A) Spatial expression patterns of GFP 288 reporter constructs containing element $\mathrm{G}$ truncations in S. purpuratus embryos at $48 \mathrm{hpf}$. (B) 289 Spatial expression patterns of GFP reporter constructs containing element $G$ truncations in $L$. 290 variegatus embryos at $28 \mathrm{hpf}$. Representative images of PMC-specific and ectopic GFP 291 expression are shown for some constructs. Top rows: GFP fluorescence. Bottom rows: GFP 292 fluorescence overlayed onto DIC images. Scale bar: $50 \mu \mathrm{m}$. 


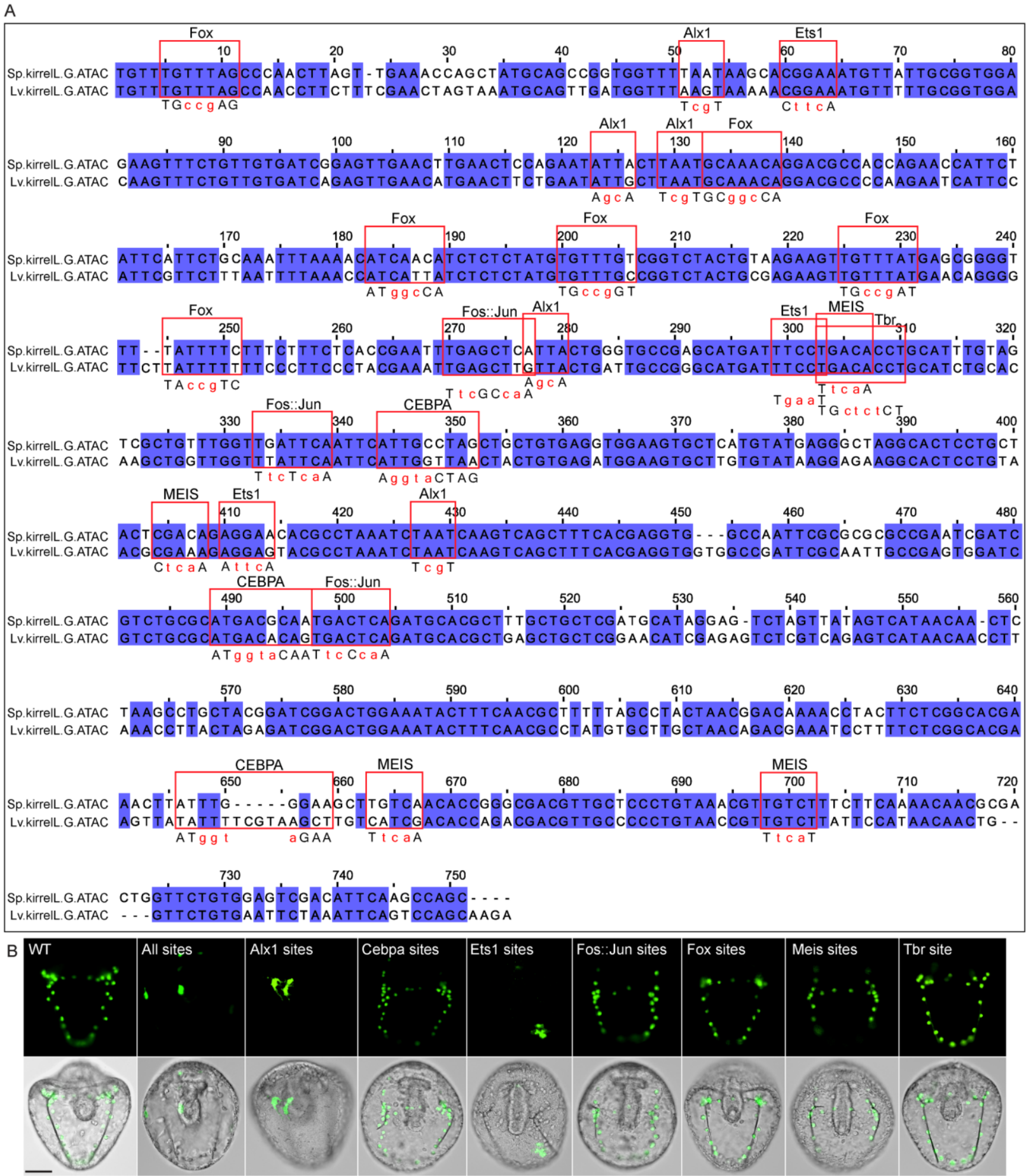

294 Figure S3: Mutational analysis of G.ATAC element. (A) Cluster Omega alignment of Sp-kirrelL and Lv-kirrelL G.ATAC sequences. Violet shading indicates conserved sequences. Red boxes highlight putative transcription factor binding sites. (B) Spatial expression pattern of GFP reporter constructs containing different G.ATAC element mutants. Top row: GFP fluorescence. Bottom row: GFP fluorescence overlayed onto DIC images. Scale bar: $50 \mu \mathrm{m}$. 

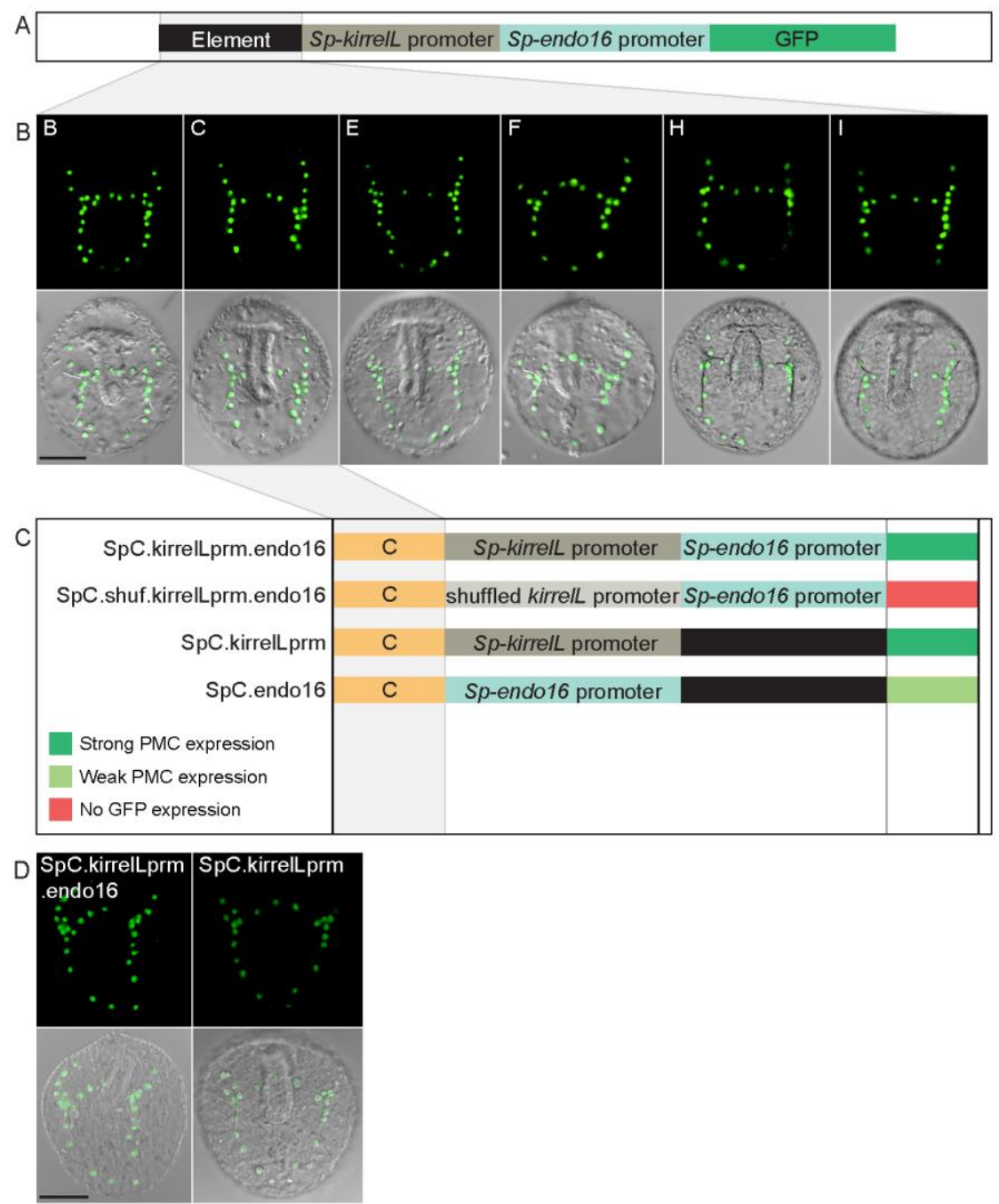

300 Figure S4: Interactions between Sp-kirrelL CREs and the endogenous Sp-kirrelL promoter. (A) Diagram showing the backbone of a modified, EpGFPll reporter construct containing the endogenous Sp-kirrelL promoter upstream of the Sp-endo16 promoter. (B) Spatial expression patterns of the modified GFP reporter constructs containing the various Sp-kirrelL elements indicated (elements B, C, E, F, H, and I). (C) Summary of GFP expression patterns of control constructs to determine whether element activity is dependent on the presence of the Sp-kirrelL promoter. Criteria for strong and weak PMC expression are defined in Figure 2. (D) Spatial expression patterns of GFP reporter constructs containing element C and the Sp-kirrelL promoter, with or without the Sp-endo16 promoter. Top rows: GFP fluorescence. Bottom rows: GFP fluorescence overlayed onto DIC images. Scale bar: $50 \mu \mathrm{m}$. 
Relative contributions of individual CREs in the context of the entire Sp-kirrelL regulatory apparatus

Our analysis identified multiple CREs in the vicinity of the Sp-kirrelL locus that were capable of driving PMC-specific reporter expression when cloned into plasmids that contained the endogenous Sp-kirrelL promoter. To explore further the relative contributions of these various elements to Sp-kirrelL expression in vivo, we examined their function in the context of the complete transcriptional control system of the gene. For these studies, we utilized a $130 \mathrm{~kb}$ bacterial artificial chromosome (BAC) that contained the single exon Sp-kirrelL gene, flanked by $65 \mathrm{~kb}$ of sequences in each direction. We used recombination-mediated genetic engineering (recombineering) to replace the single Sp-kirrelL exon seamlessly with either GFP or mCherry coding sequence (Figure 5A). We found that Sp.kirrelL.GFP.BAC faithfully recapitulated the expression of endogenous Sp-kirrelL in the PMCs at $48 \mathrm{hpf}$ with minimal ectopic expression (Figure S5). We next generated deletion mutants based on results from our plasmid GFP reporter assays to quantitatively assess the contributions of elements $A$ to $\mathrm{G}$ to $S p$-kirrelL transcriptional regulation. We found that deletion of elements $A$ to $G$ ( $\triangle$ CRE.GFP.BAC) completely abolished GFP expression. We also observed that retaining the minimal endogenous $S p$-kirrell promoter ( $\triangle$ CRE.kirrelLprm.GFP.BAC) did not rescue GFP expression, demonstrating that elements A to $\mathrm{G}$ are necessary for $S p$ kirrelL expression in the context of the Sp.kirrelL.GFP.BAC consistent with our previous, plasmid-based analysis.

To directly compare the spatial expression patterns of deletion mutants with that of the parental BAC, we generated BAC mutants containing deletion of individual elements and co-injected them into fertilized eggs with a parental mCherry BAC. We found that a BAC containing deletion of the element G ( $\triangle$ G.GFP.BAC, which included a deletion of the Sp-kirrelL promoter) abolished GFP expression at $48 \mathrm{hpf}$ (Figure 5B and Figure S5). By contrast, deletion of all of element $\mathrm{G}$ except for the promoter region ( $\triangle$ G.kirrelLpromoter.GFP.BAC), resulted in a GFP spatial expression pattern similar to that of the parental mCherry. These findings confirmed the importance of the Sp-kirrell promoter in supporting PMC-specific expression of the gene and showed that this region is essential even when all distal CREs are present. BACs containing individual deletions of other elements all remained active at $48 \mathrm{hpf}$ and supported PMC-specific reporter expression, pointing to considerable redundancy in the contribution of each element to Sp-kirrell expression.

To examine the relative contribution of distal CREs more rigorously, we measured levels of reporter transcripts using a NanoString nCounter. For each mutant BAC, we coinjected embryos with mCherry tagged, parental BAC and the GFP-tagged, mutant BAC and quantified the expression level of each reporter gene at four time points $(20,30,50$, and $65 \mathrm{hpf}$ ) (Figure 5C and Supplemental Table S2). We found that deletion of element 
349 C resulted in approximately a $50 \%$ reduction in expression compared to WT BAC. As we 350 observed previously, GFP expression was completely abolished when element G was 351 deleted ( $\triangle$ G.GFP.BAC) and this effect was diminished when the Sp-kirrelL promoter was 352 retained ( $\triangle$ G.kirrelLprm.GFP.BAC). Quantitative analysis revealed, however, that 353 retention of the $S p$-kirrelL promoter alone resulted in only a partial rescue of expression, 354 with overall levels reduced substaintially compared to the wild-type BAC. We also 355 observed that deletion of element $\mathrm{H}$ resulted in decreased expression levels. Taken 356 together, our qualitative and quantitative analyses show that at early stages of embryo 357 development, Sp-kirre/L expression is controlled by multiple CREs, notably the C, G, and $358 \mathrm{H}$ modules, acting in concert with the Sp-kirrelL promoter. 

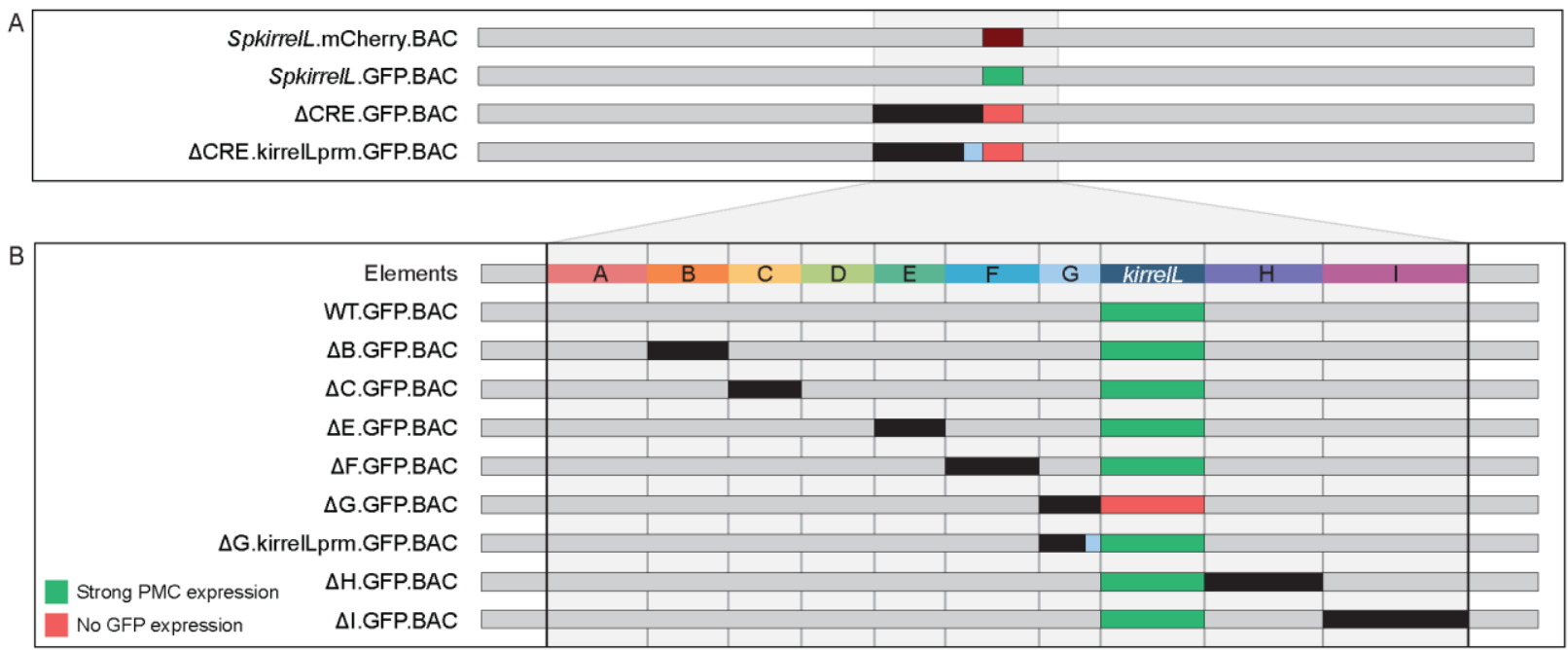

C
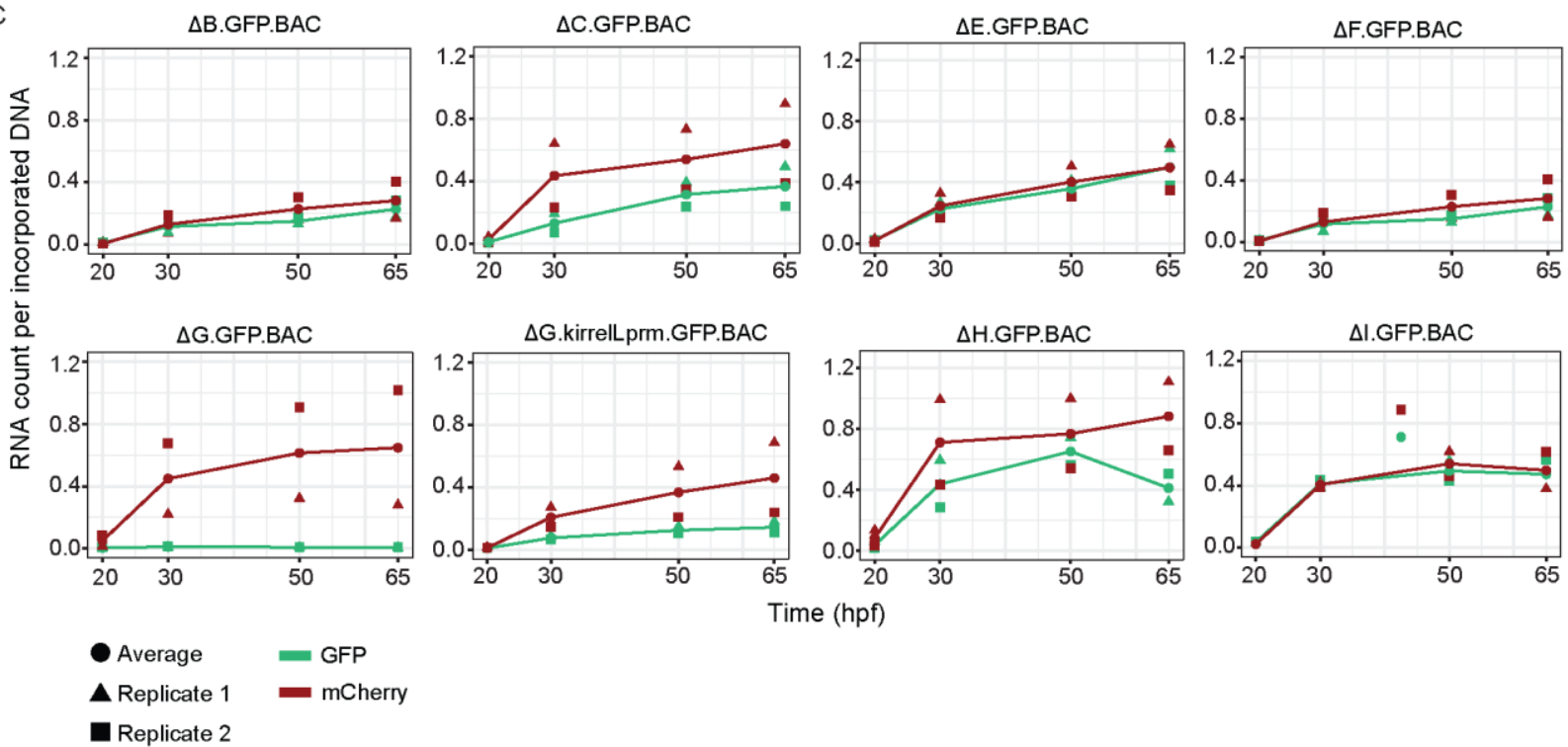

360 Figure 5: Sp-kirrelL cis-regulatory analysis using BACs. (A) BAC deletions show that elements A-G are necessary for GFP expression, regardless of the presence of the endogenous Sp-kirrelL core promoter elements. (B) Summary of GFP expression patterns of individual Sp-kirrelL elements using GFP BAC deletions. Criteria for strong PMC expression are defined in Figure 2. (C) Quantitative NanoString analysis of reporter expression in embryos co-injected with parental mCherry and mutant GFP BACs. Embryos were collected at 20, 30, 50, and $65 \mathrm{hpf}$. The average expression profile for each pair of BAC injection was calculated from NanoString counts of two biological replicates (see Materials and Methods). 

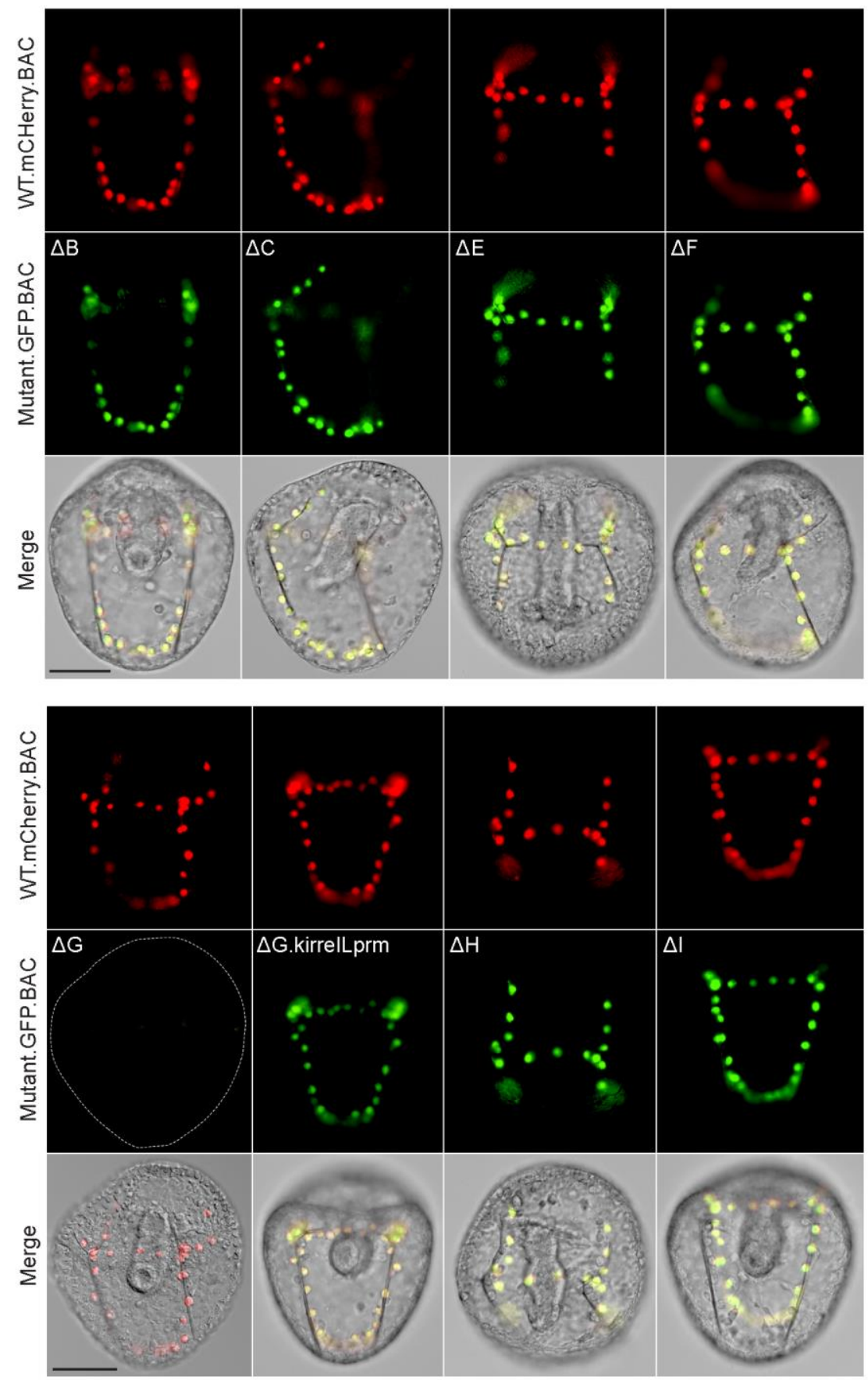

369 Figure S5: Spatial expression patterns of embryos co-injected with parental mCherry and mutant 370 GFP BACs. Top rows: mCherry fluorescence. Middle rows: GFP fluorescence. Bottom rows: 371 mCherry and GFP fluorescence overlayed onto DIC images. Scale bar: $50 \mu \mathrm{m}$. 
Cross-species analysis of echinoderm kirrelL CREs

373

374

375

376

377

378

379

380

381

382

383

384

385

386

387

388

389

390

391

392

393

394

395

396

397

398

399

As the non-coding region directly upstream of the translational start site of $\mathrm{Sp}$ kirrelL was found to contain transcriptional control elements, we asked whether sequences directly upstream of kirrelL genes from other echinoderm classes might contain conserved CREs that have activity in S. purpuratus PMCs. We cloned non-coding sequences upstream of kirrell genes from Eucidaris tribuloides (pencil urchin), Parastichopus parvimensis (sea cucumber), Patiria miniata (sea star), Acanthaster planci (crown-of-thorns starfish), Ophionereis fasciata (brittle star), and Anneissia japonica (feather star) into the EpGFPII plasmid and injected them into fertilized $S$. purpuratus eggs (Figure 6A). Remarkably, we found that all six drove GFP expression in sea urchin embryos, with five out of six exhibiting strong GFP expression selectively in PMCs (Figure 6B). Taken together, these observations indicate that kirrelL CREs across echinoderm species are highly conserved. We found it particularly striking that kirrelL CREs from deeply divergent echinoderm species that do not form embryonic or larval skeletons (sea stars and feather stars) drive PMC-selective GFP expression in sea urchin embryos.

Although KirrelL has been shown to be an important morphoeffector gene in the sea urchin embryo, where it plays an essential role in PMC-PMC fusion, its expression in adult sea urchins has not been examined. We observed Lv-kirrelL expression in the skeletogenic centers of the adult rudiment and in the spine of five-week-old juvenile sea urchins (Figure 6B). The expression pattern of $L v$-kirrel $L$ was very similar to that of $L v$ msp130r2, a highly expressed biomineralization gene (Figure S6B). In contrast, expression of $P m$-kirrel $L$ was not detected during early embryonic and larval development in P. miniata, which does not from a larval skeleton (Figure 6C). Pm-kirrelL is, however, expressed in the developing adult rudiment in pre-metamorphic, late-stage sea star larva and in the adult skeletogenic centers in juvenile sea stars (Figure 6D). As a control, we showed Pm-ets 1 expression in the mesenchyme cells during early development and an expression pattern in the adult rudiment and skeletogenic centers in juvenile sea stars that closely resembled that of Pm-kirrelL (Figure S6C). 

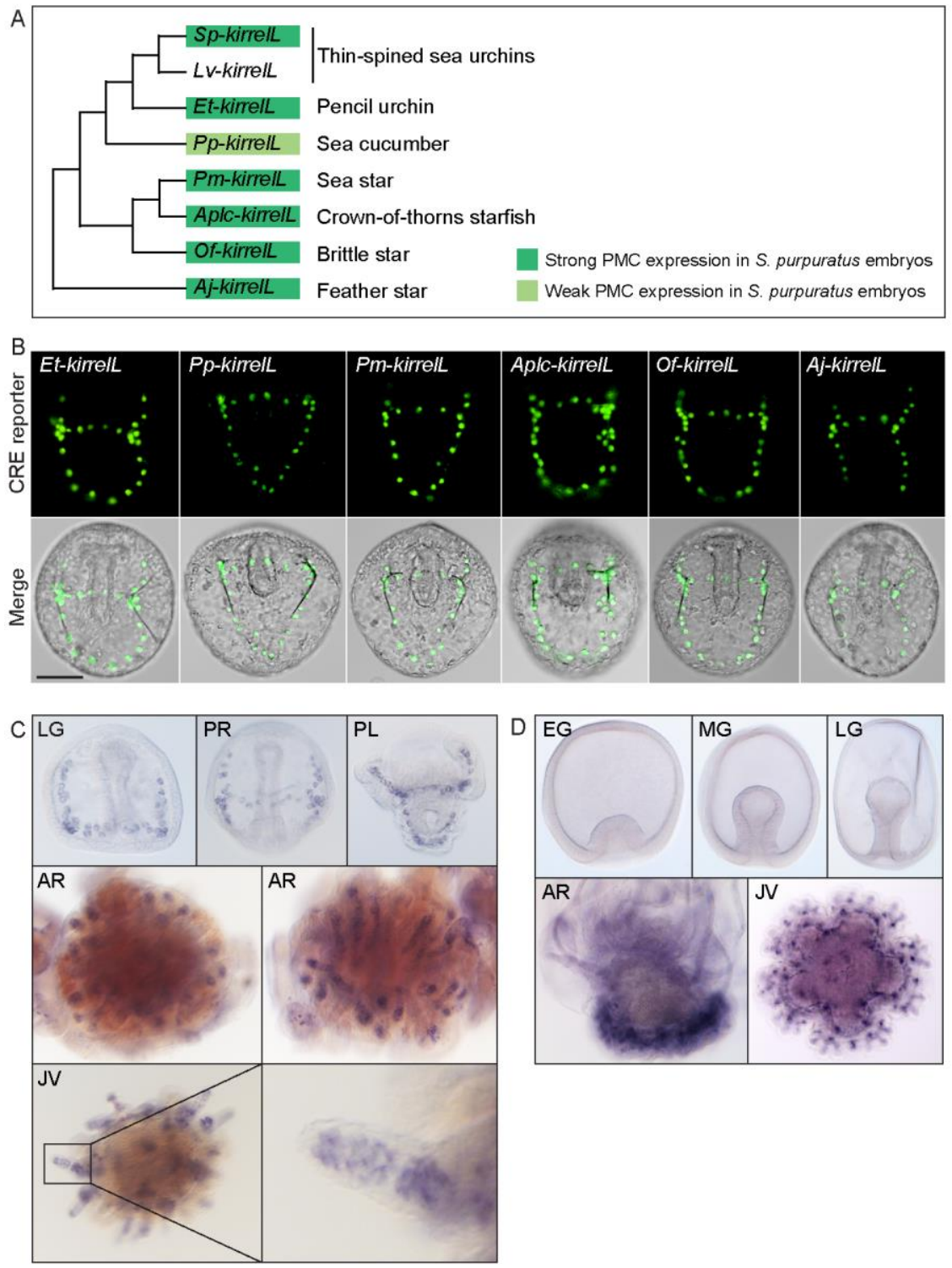

Figure 6: Cross-species analysis of kirrelL CREs from diverse members of the echinoderm phylum. (A) Phylogenetic relationships of kirrell genes based on the consensus view of evolutionary relationships among echinoderms. Branch lengths are not drawn to scale. Box colors correspond to expression of GFP in $S$. purpuratus embryos, driven by non-coding sequences upstream of kirrell genes of Eucidaris tribuloides (Et-kirrelL), Parastichopus parvimensis (PpkirrelL), Patiria miniata (Pm-kirrelL), Acanthaster planci (Aplc-kirrelL), Ophionereis fasciata (OfkirrelL), and Anneissia japonica (Aj-kirrelL). Criteria for strong and weak PMC expression are defined in Figure 2. (B) Spatial expression patterns of GFP reporter constructs containing kirrelL CREs from other echinoderm species in $S$. purpuratus embryos at $48 \mathrm{hpf}$. Top row: GFP fluorescence. Bottom row: GFP fluorescence overlayed onto DIC images. Scale bar: $50 \mu \mathrm{m}$. (C) 411 Representative WMISH images showing Lv-kirrelL expression during $L$. variegatus development. (D) Pm-kirrelL expression during P. miniata development. EG, early gastrula; MG, mid-gastrula; LG, late gastrula; PR, prism stage; PL, pluteus larva; AR, adult rudiment; JV, juvenile stage. 

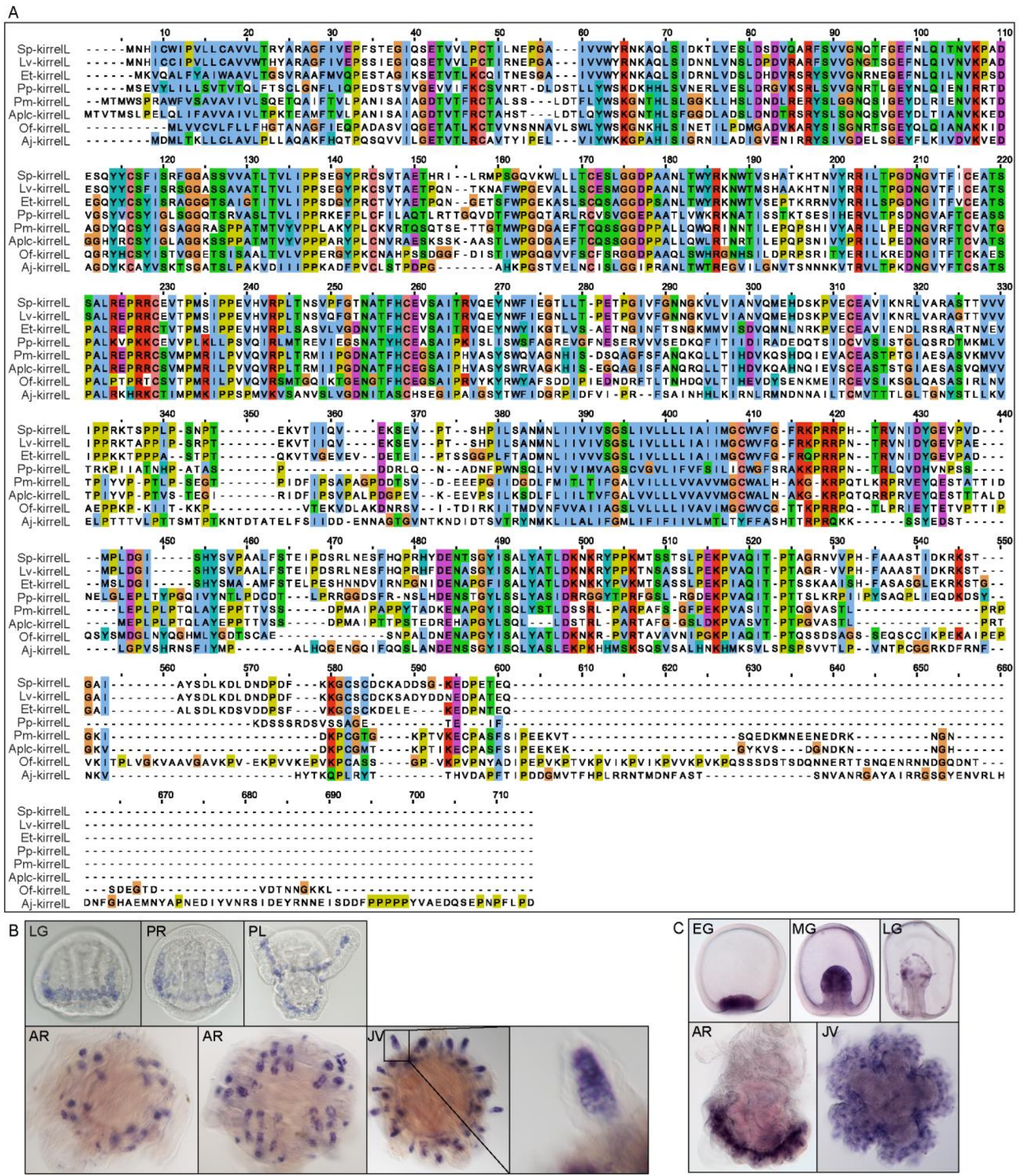

Figure S6: Alignment of echinoderm KirrelL proteins and representative WMISH images of positive control probes. (A) Clustal alignment of echinoderm KirrelL proteins from Eucidaris

417 tribuloides (Et-kirrelL), Parastichopus parvimensis (Pp-kirrelL), Patiria miniata (Pm-kirrelL), 418 Acanthaster planci (Aplc-kirrelL), Ophionereis fasciata (Of-kirrelL), and Anneissia japonica (Aj419 kirrelL). Colors correspond to the Clustal default residue coloring scheme. (B) Lv-msp130r2 420 expression during $L$. variegatus development. (C) Pm-ets1 expression during $P$. miniata 
421 development. EG, early gastrula; MG, mid-gastrula; LG, late gastrula; PR, prism stage; PL, 422 pluteus larva; AR, adult rudiment; JV, juvenile stage.

423

424

425

426

427

428

429

430

431

432

433

434

435

436

437

438

439

440

441

442

443

444

445

446

447

448

\section{Dissection of a candidate adult skeletogenic CRE}

As sea stars do not form a larval skeleton but express kirrelL specifically in adult skeletogenic centers, we exploited the activity of the Pm-kirrelL CRE in sea urchin embryos as a potential proxy for identifying transcriptional inputs that ordinarily control this gene in adult echinoderms (see Discussion). We performed truncations and mutations of the regulatory regions upstream of the Pm-kirrelL gene to identify direct transcriptional inputs (Figure 7). Subdivision of the $\sim 4 \mathrm{~kb}$ Pm-kirrelL regulatory region showed that activity was restricted to the proximal region (Pm2), and further analysis revealed that a 614 bp region (PmG) that was sufficient to drive strong PMC-specific GFP expression in $S$. purpuratus embryos (Figure 7B and Figure S7A). Phylogenetic footpriting of genomic sequences from $P$. miniata and the closely related crown-of-thorns starfish (A. planci) showed substantial similarity in this region (Figure 7A). We performed mutational analysis of the PmG element and found that this CRE receives positive inputs from both Alx1 and Ets1 (Figure 7C and Figure S7B), similar to the Sp-kirrelL C and G.ATAC elements. We next asked whether PmG1 and PmG2 elements, which are located near the Pm-kirrelL transcriptional start site, could interact with distal Sp-kirrelL elements, thereby substituting for the endogenous $S p$-kirrelL promoter. For this analysis, we generated chimeric EpGFPII reporter constructs that contained the sea urchin SpkirrelL element $\mathrm{C}(\mathrm{SpC})$ adjacent to the sea star PmG1 or PmG2 element (Figure 7D). We found that PmG1 and PmG2 were both interchangeable with the Sp-kirrelL promoter and that interactions between SpC and PmG1 or PmG2 supported strong PMC-specific GFP expression in $S$. purpuratus embryos (Figure S7C). In a construct containing a PmG2 element with shuffled sequence, GFP expression was abolished. These observations highlight a striking conservation of sequence and function in kirrell promoters from deeply divergent echinoderm species. 

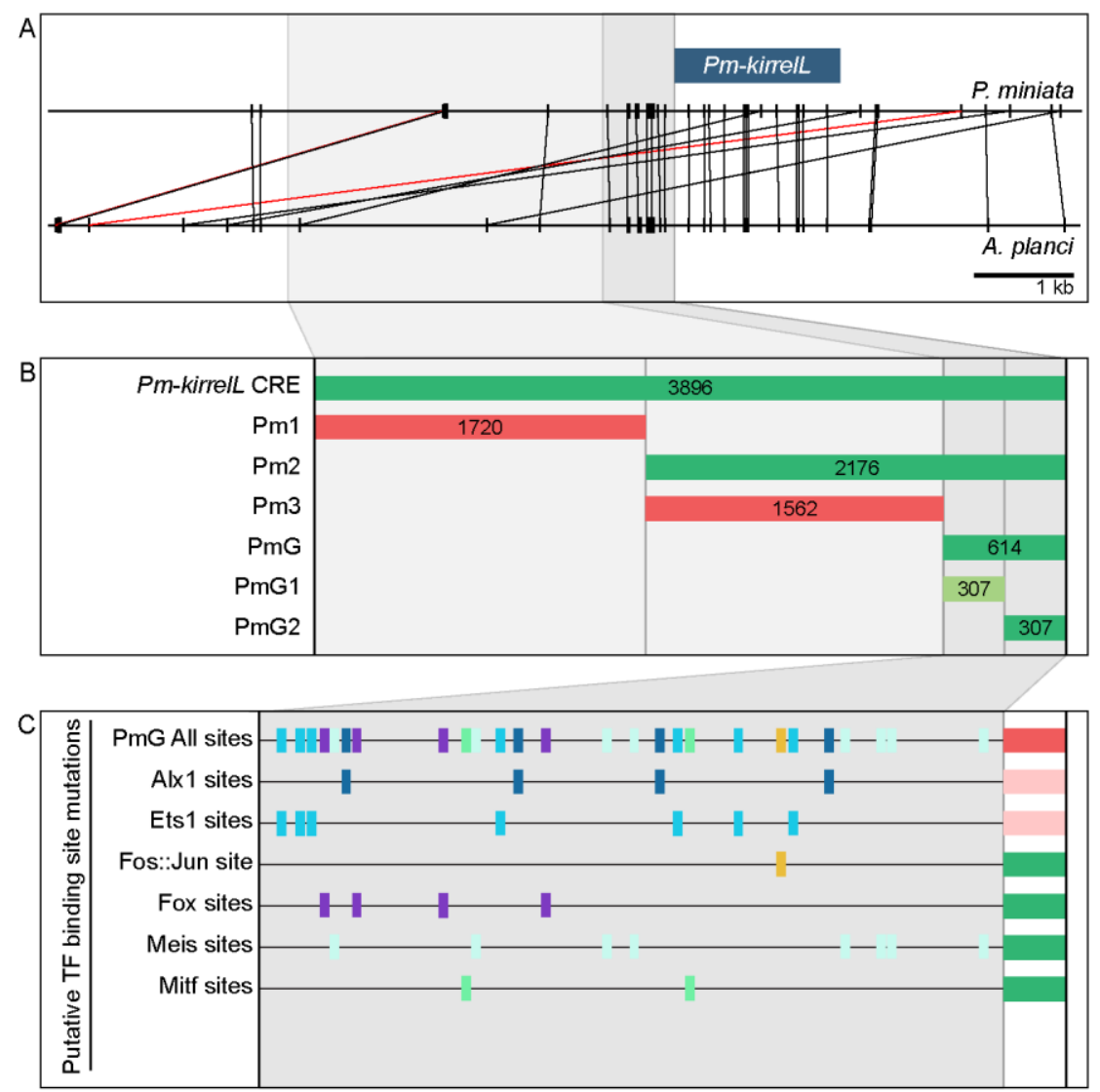

\begin{tabular}{|c|c|c|c|}
\hline SpC.PmG1 & C & PmG1 & endo16 promoter \\
\hline SpC.PmG2 & C & PmG2 & endo16 promoter \\
\hline SpC.shuf.PmG1 & C & shuffled PmG1 & endo16 promoter \\
\hline \multirow[t]{5}{*}{ SpC.shuf.PmG2 } & C & shuffled PmG2 & endo16 promoter \\
\hline & \multicolumn{3}{|c|}{ Strong PMC expression } \\
\hline & \multicolumn{3}{|c|}{ Weak PMC expression } \\
\hline & \multicolumn{3}{|c|}{ No GFP expression } \\
\hline & \multicolumn{3}{|c|}{ Ectopic expression } \\
\hline
\end{tabular}

Figure 7: Functional analysis of non-coding genomic sequences upstream of Pm-kirrelL to identify CREs. (A) Phylogenetic footprinting of genomic sequences near $P$. miniata and $A$. planci kirrelL using GenePalette. Black lines indicate identical sequences of 15 bp or longer in the same orientation while red lines indicate identical sequences of $15 \mathrm{bp}$ or longer in the opposite orientation. (B) Summary of GFP expression regulated by non-coding sequences upstream of the Pm-kirrelL translational start site. (C) Summary of GFP expression driven by PmG element mutants. (D) Summary of GFP expression regulated by chimeric reporter constructs containing Sp-kirrelL element C and Pm-kirrelL G1 or G2 elements. Criteria for strong and weak PMC expression are defined in Figure 2. Ectopic expression is defined as $>10 \%$ of total injected embryos expressing GFP, with majority exhibiting expressing in cells other than PMCs. 

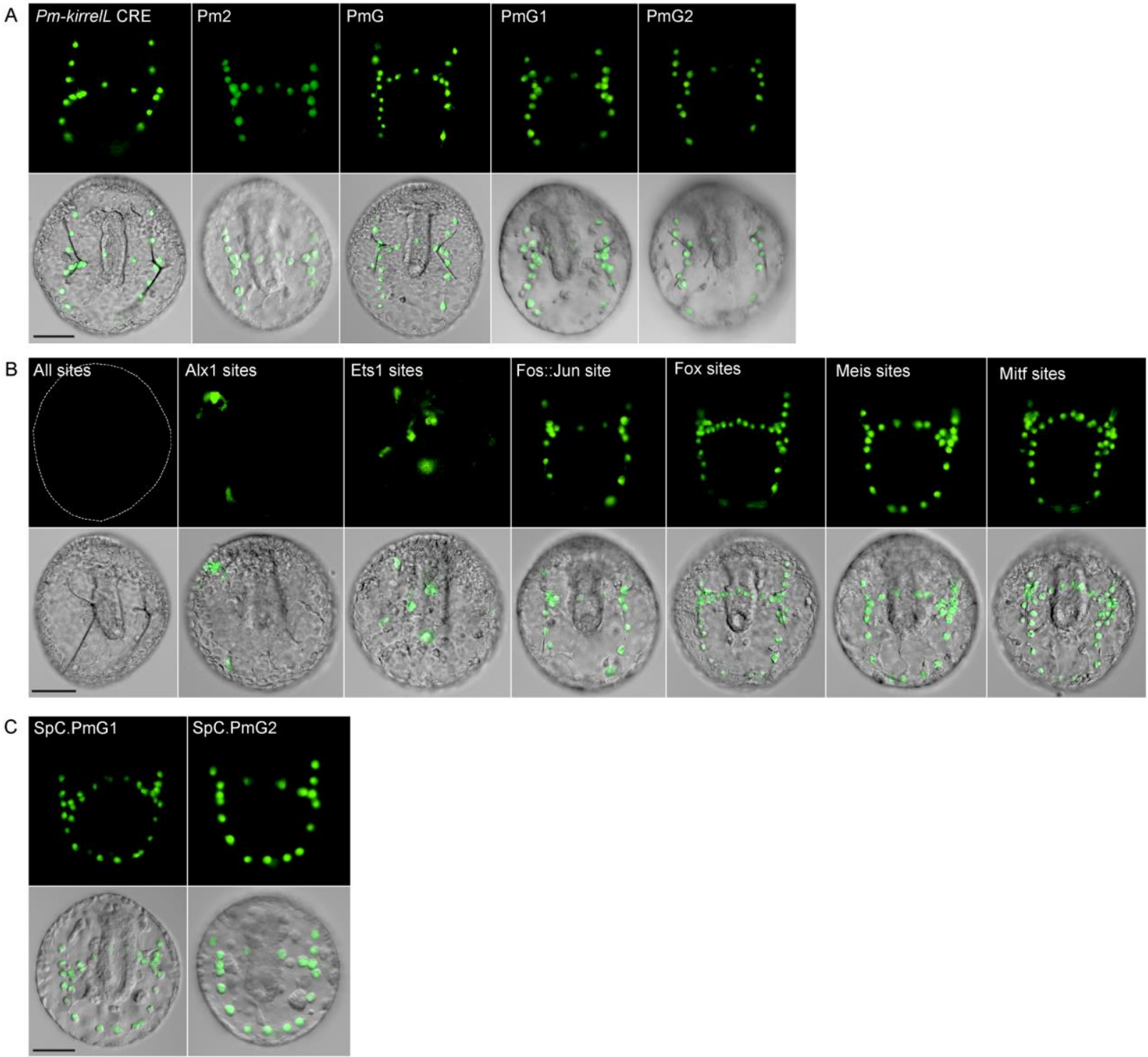

Figure S7: Sea star Pm-kirrelL CRE truncation and mutational analysis. (A) Spatial expression patterns of GFP reporter constructs containing Pm-kirrelL truncations in $S$. purpuratus embryos at $48 \mathrm{hpf}$. (B) Spatial expression patterns of GFP reporter constructs containing sea star PmG element mutants in $S$. purpuratus embryos at $48 \mathrm{hpf}$. (C) Spatial expression patterns of chimeric reporter constructs containing Sp-kirrelL element C and Pm-kirrelL G1 or G2 elements. Top rows: GFP fluorescence. Bottom rows: GFP fluorescence overlayed onto DIC images. Scale bar: 50 $\mu \mathrm{m}$. 


\section{Discussion}

Linking developmental GRNs to morphogenesis

Recent studies with echinoderms have elucidated the architecture of developmental GRNs, including the GRN deployed specifically in embryonic skeletogenic mesenchyme of sea urchins (Shashikant et al., 2018a). Although these studies have focused largely on interactions among regulatory genes that constitute the core of such networks, the importance of GRNs from a developmental perspective is that they underlie the dramatic anatomical changes that characterize embryogenesis (Ettensohn, 2013; Smith et al., 2018). In that respect, GRNs have considerable power in explaining the transformation of genotype into phenotype. Moreover, if GRNs are to be useful in understanding the evolution of morphology, currently a major goal of comparative GRN biology, the developmental mechanisms by which these genetic networks drive morphology must be addressed. This work seeks to partially fill this conceptual gap by elucidating the transcriptional control of $S p$-kirrelL, an effector gene required for cell-cell fusion, an important morphogenetic behavior of PMCs.

\section{The cis-regulatory apparatus of Sp-kirrelL}

The combinatorial control of CRE function is important for driving complex gene expression patterns during animal development. In the present study, we identified key regulatory elements and transcription factor inputs that control Sp-kirrelL expression. Using plasmid reporter constructs, we identified 7 CREs (elements B, C, E, F, G, H, and I) that were individually sufficient to drive strong PMC-specific GFP expression when placed adjacent to the native Sp-kirrelL promoter. Most of these same elements failed to drive reporter expression at detectable levels, however, when cloned directly adjacent to the 140 bp Sp-endo16 core promoter, a component of EpGFPII, a vector widely used for cis-regulatory analysis in sea urchins. As proximal promoter elements have been shown to tether more distal elements in other organisms (Calhoun et al., 2002), we hypothesize that such tethering activity is present in the $301 \mathrm{bp}$ Sp-kirrelL promoter element contained in element $\mathrm{G}$. Tethering activity would also account for the fact the regulatory sites in the C element (i.e., those contained in C.ATAC and C.ChIP) must be in close proximity to the Sp-endo16 promoter to activate transcription, while these same sites can function at a greater distance when working in concert with the endogenous Sp-kirrelL promoter. These findings highlight the potential limitations of transgenic reporter assays that rely exclusively on exogenous and/or core promoters.

As multiple CREs were capable of supporting PMC-specific reporter expression in combination with the Sp-kirrelL promoter, we performed BAC deletion analysis to determine the relative contributions of these elements to Sp-kirrelL expression. We 
quantified reporter expression using a newly developed, Nanostring-based assay that allowed us to measure the extent of transgene incorporation and reporter expression. We found that a single deletion of elements A through $G$ entirely abolished GFP expression, even in the presence of the native $S p$-kirrelL promoter, pointing to this region as the major regulatory apparatus of the gene and demonstrating that any CREs outside this region (including elements $\mathrm{H}$ and I) are insufficient to support transcription during embryogenesis. Consistent with plasmid reporter assays, our quantitative BAC analysis confirmed that elements $\mathrm{C}$ and $\mathrm{G}$ both make major contributions to $\mathrm{Sp}$-kirrelL expression. Furthermore, we confirmed that the $S p$-kirrell native promoter is required for BAC reporter activity, also consistent with our plasmid reporter assays and with the hypothesis that the CREs are brought into physical contact with the promoter by chromatin looping during transcription. We observed that deletion of element $\mathrm{H}$, which consisted of the $S p$ kirrelL 3'-UTR, also resulted in decreased expression of the BAC reporter at 30 and 65 hpf. Although an exogenous polyadenylation site was inserted at the 3 ' end of the reporter coding sequence during BAC recombineering and was therefore present in ftablall constructs, we cannot exclude the possibility that transcription extended beyond this site and that deletion of the 3'-UTR influenced the processing or stability of the Sp-kirrelL transcript rather than transcription.

Elements B, E, F, and I each drove PMC-specific reporter expression in plasmid constructs that contained the Sp-kirrelL promoter, but deletion of these elements individually from the $S p$-kirrelL BAC did not quantitatively affect reporter expression at the developmental stages we examined. There are several possible explanations for this. First, these CREs may have no regulatory function in vivo. According to this view, the transcriptional activity of these elements in plasmid constructs was an artifact of bringing them in close proximity to the native Sp-kirrelL promoter. This view is inconsistent, however, with the fact that most of these elements $(B, E$, and $I)$ contain other signatures of enhancer activity. All three elements are hyper-accessible in PMCs relative to other cell types at $24 \mathrm{hpf}$ as assayed by ATAC-seq, and elements $\mathrm{E}$ and I are also associated with eRNA signal during early development (Fig. 1). Moreover, these elements exhibited some degree of promoter specificity in our reporter assays; i.e., they were active in combination with the Sp-kirrelL promoter but not the Sp-endo16 core promoter. These findings suggest that some or all of these elements ordinarily have a regulatory function. They may modulate the precision of $S p$-kirrelL expression during early development in subtle ways that our assays did not detect (Lagha et al., 2012) or they may be entirely redundant; i.e., deletion of any one of these elements may result in the complete assumption of its function by other elements. This might be the case, for example, if functionally equivalent CREs ordinarily share the Sp-kirrell promoter. Lastly, although these elements are associated with eRNA expression and cell type-specific accessibility early in embryogenesis, their primary function may be to regulate $S p$-kirrelL expression during stages of development later than those assayed in this study. 


\section{Co-regulation of elements $\mathrm{C}$ and $\mathrm{G}$ by $\mathrm{Alx} 1$ and Ets 1}

The results of both plasmid- and BAC-based reporter assays showed that elements $\mathrm{C}$ and $\mathrm{G}$ provide crucial inputs into Sp-kirrelL. Detailed dissection of these key elements identified consensus Ets 1 and Alx1 binding sites that were essential for activity. This finding was consistent with previous evidence that perturbation of alx1 or ets 1 function using antisense morpholinos results in a dramatic reduction of $S p$-kirrelL expression (Rafiq et al., 2014). Moreover, ChIP-seq studies have shown that Alx1 binds directly to both elements (Khor et al., 2019). We cannot, however, exclude the possibility that other ETS and homeodomain family members expressed in PMCs (e.g., Erg and Alx4) also bind to these sites. Interestingly, although paired-class homeodomain proteins (including Alx1-related proteins found in vertebrates) are thought to regulate transcription primarily through their binding to palindromic sites that contain inverted TAAT sequences (e.g., ATTANNNTAAT), we identified a half site (ATTA) in element $C$ that was required for activity. This finding supports other recent work which has shown that half sites play a more prominent role in the transcriptional activity of Alx1 than was previously appreciated (Guerrero-Santoro et al., 2021).

Based on gene knockdown studies and the epistatic gene relationships they reveal, Oliveri et al. (2008) proposed that several PMC effector genes are regulated through a feed-forward circuit involving Alx1 and Ets1. They showed that Ets1 positively regulates al $x 1$ and that both regulatory inputs are necessary to drive expression of several biomineralization-related genes. Our findings support such a model and extend it by demonstrating that the topology of this feed-forward regulation is very simple- both Alx1 and Ets1 provide direct, positive inputs into CREs associated with Sp-kirrelL. We identified dual, direct inputs into two different CREs, one associated with the promoter (element G) and a more distal element (element C). Evidence from other recent studies suggest that direct co-regulation by Alx1 and Ets1 is a widespread mechanism for controlling PMC effector gene expression. Genome-wide analysis of Sp-Alx1 ChIP-seq peaks located near effector gene targets showed that both Alx1 and Ets1 consensus binding sites were highly enriched in these regions (Khor et al., 2019) and both Alx1 and Ets 1 binding sites are enriched in regions of chromatin that are hyper-accessible in PMCs relative to other cell types (Shashikant et al., 2018b). Our analysis of Sp-kirrelL reveals that feed-forward regulation by Alx1 and Ets1 controls not only the expression of biomineralization-related genes but also genes that regulate PMC behavior, thereby integrating these cellular activities.

Davidson (1986) proposed that sea urchins, ascidians, nematodes, and several other animal groups develop by a so-called "Type I" mechanism, a mode of development characterized by the early embryonic expression of terminal differentiation genes. A prediction of this model is that Type I embryos deploy developmental GRNs that are relatively shallow; i.e., there are few regulatory layers between cell specification and cell 
differentiation. The cis-regulatory control of Sp-kirrelL by Alx1 and Ets1 supports this prediction; both transcription factors are activated during early embryogenesis and provide direct, positive inputs into Sp-kirrelL. Although mutations of other putative transcription factor binding sites in elements $C$ and $G$ did not result in any noticeable effects on reporter expression in our studies, it should be noted that perdurance of GFP mRNA or protein following activation by early regulatory inputs such as Alx1 and Ets1 might have masked effects of such mutations on later stages of embryogenesis.

\section{Evolutionary conservation of echinoderm kirrelL CREs}

All adult echinoderms have elaborate, calcitic endoskeletons, but larval skeletal elements are found only in echinoids, ophiuroids, and holothuroids (the latter form only a very rudimentary larval skeleton). It is widely believed that the adult skeleton was present in the most recent common ancestor of all echinoderms and that larval skeletons arose subsequently through a developmental re-deployment of the adult program (see reviews by Cary and Hinman, 2017; Koga et al., 2014; Shashikant et al., 2018a). It is debated, however, whether this re-deployment occurred only once, with a subsequent loss of larval skeletons in asteroids, or more than once, with larval skeletons appearing independently in several groups. Our studies establish kirrelL as a component of the ancestral echinoderm skeletogenic GRN, which also included alx1, ets1, and vegfr-10-lg (Erkenbrack and Thompson, 2019; Shashikant et al., 2018a).

There is abundant evidence that mutations in cis-regulatory sequences contribute to phenotypic evolution (Rebeiz and Tsiantis, 2017; Wray, 2007). At the same time, there are examples of evolutionarily conserved GRN topologies and transcription factor binding sites, often between relatively recently diverged taxa (e.g., mice and humans) but sometimes more deeply conserved (Rebeiz et al., 2015). In the present study, we showed that non-coding sequences upstream of the translational start sites of kirrelL genes from a diverse collection of echinoderms supported PMC-specific reporter expression in sea urchin embryos. These echinoderms included a crinoid ( $A$. japonica) and two sea stars (A. planci and $P$. miniata), taxa that diverged from echinoids 450-500 million years ago (Paul and Smith, 1984; Pisani et al., 2012). The deep evolutionary separation of these groups reveals a remarkable conservation of the kirrelL regulatory apparatus over this vast time period. Although the amino acid sequences of KirrelL proteins are well conserved within the phylum (Figure S6A), the sequences of the upstream regulatory regions we identified are more divergent. Despite limited nucleotide sequence conservation, dissection of the $P m$-kirrelL regulatory region provided evidence that in sea stars, as in sea urchins, Alx1 and Ets1 provide direct, positive inputs into kirrelL. Moreover, we showed that regulatory elements directly upstream of the Pm-kirrelL translation start site could substitute for the native Sp-kirrelL promoter in supporting the 
622 activity of the $S$. purpuratus $\mathrm{C}$ element, an effect that we hypothesize reflects a deep conservation of the binding sites and proteins that mediate CRE-promoter tethering.

624

625

626

627

628

629

630

631

632

633

634

635

636

637

638

639

640

641

642

643

644

645

646

647

648

649

The embryonic skeletogenic GRN of sea urchins has been elucidated in considerable detail, but analysis of the ancestral, adult program has this far been limited to comparative gene expression studies, as there are several technical hurdles to molecular perturbations of adult echinoderms. Because sea stars do not express kirrell at embryonic stages and lack a larval skeleton, but express kirrell in adult skeletogenic centers, we conclude that the function of the sea star kirrelL cis-regulatory system is to control the transcription of this gene in the adult. Thus, our identification of Alx 1 and Ets 1 inputs into the Pm-kirrelL regulatory region provides evidence that these inputs are required in skeletal cells of the adult sea star, consistent with the finding that both Ets 1 and Alx1 are expressed selectively by these cells (Gao and Davidson, 2008). We cannot exclude the possibility that the regulatory interactions we detected in the context of the $S$. purpuratus embryo are vestiges of an ancient, larval skeletogenic program that has since been lost in sea stars, if indeed this was the evolutionary trajectory of larval skeletogenesis within echinoderms. This interpretation, however, would require the evolutionary conservation of the relevant regulatory DNA sequences over a vast period of time despite their complete lack of function, a scenario that seems very unlikely. We propose instead that our findings provide the first glimpse of functional gene interactions in the ancestral, adult echinoderm skeletogenic program and highlight the remarkable conservation of this program in adults and embryos. They strongly support the view that co-option of the adult skeletogenic GRN into the embryo occurred, at least in part, via a heterochronic shift in the expression of Alx1 and Ets1. This would have been sufficient to transfer a large part of the skeletogenic GRN into the embryo, as the transcription of many key effector genes, including kirrelL, was already directly linked to Alx1 and Ets1 expression. Direct analysis of CRE structure and function in the adult skeletogenic centers of sea stars and sea urchins will be required to more fully elucidate the architecture of the ancestral network. 
bioRxiv preprint doi: https://doi.org/10.1101/2021.08.27.457814; this version posted August 28, 2021. The copyright holder for this preprint (which was not certified by peer review) is the author/funder, who has granted bioRxiv a license to display the preprint in perpetuity. It is made available under aCC-BY 4.0 International license.

\section{Supplemental tables}

651 Supplemental Table S1: Quantification of GFP expression patterns in embryos injected 652 with reporter constructs.

653 Supplemental Table S2: Summary of NanoString analysis.

654 Supplemental Table S3: NanoString analysis probe target sequences. 


\section{Materials and Methods}

Animals

Adult Strongylocentrotus purpuratus and Patiria miniata were acquired from Patrick Leahy (California Institute of Technology, USA). Adult Lytechinus variegatus were acquired from the Duke University Marine Laboratory (Beaufort, NC, USA) and from Pelagic Corp. (Sugarloaf Key, FL, USA). Spawning of gametes was induced by intracoelomic injection of $0.5 \mathrm{M} \mathrm{KCl}$. $S$. purpuratus and $P$. miniata embryos were cultured in artificial seawater (ASW) at $15^{\circ} \mathrm{C}$ in temperature-controlled incubators while $L$. variegatus embryos were cultured at $19-24^{\circ} \mathrm{C}$. Late-stage $L$. variegatus and $P$. miniata larvae were fed with Rhodomonas lens algae, accompanied by water changes every other day.

Generation of cis-regulatory reporter constructs

Phylogenetic footprinting between echinoderm kirrelL loci was performed using GenePalette with a sliding window size of $15 \mathrm{bp}$. GFP reporter constructs were generated by cloning putative CREs into the EpGFPII plasmid, which contains the basal promoter of Sp-endo16 (Cameron et al., 2004). Putative Sp-kirrell CREs were amplified from $S$. purpuratus genomic DNA using primers with restriction site overhangs (see Supplemental Table S3). CREs with mutations of putative transcription factor binding sites and putative CREs from echinoderm species were synthesized as gBlock gene fragments with flanking restriction sites by Integrated DNA Technologies (Coralville, IA, USA). Sequences of putative CREs from echinoderm species (other than sea urchins) were located 2-3 kb upstream of the kirrelL gene translational start sites.

\section{BAC recombineering}

Sp-KirrelL BAC-GFP reporter constructs were generated from a parental BAC (R3-28J1014544) according to established recombineering protocols (Buckley et al., 2018). The recombineering cassettes were synthesized by Integrated DNA Technologies (Coralville, IA, USA). The cassettes contained GFP coding sequence, SV40 terminator sequence, a kanamycin resistance gene between two flippase recognition target (FRT) sites and flanking homologous arms. The recombineering cassettes were transformed into EL250 cells carrying the parental BAC (pBACe3.6 vector harboring Sp-kirrelL and flanking genomic sequences) and recombinase genes were de-repressed via heat shock. EL250 cells with recombinant BACs were selected based on kanamycin resistance. To remove the kanamycin resistance gene, expression of flippase (flp) recombinase enzyme was induced using L-(+)-arabinose and colonies with the kanamycin resistance gene removed were identified by replica plating. BACs without kanamycin resistance gene were subsequently electroporated and propagated in $\mathrm{DH} 10 \beta$ cells.

DNA microinjection 
691 Microinjection of reporter constructs was performed following established protocols 692 (Arnone et al., 2004). Prior to injection, reporter constructs were linearized and mixed with 693 carrier DNA that was prepared by overnight Hindlll digestion of $S$. purpuratus or $L$. 694 variegatus genomic DNA. BAC and plasmid constructs were linearized with Ascl and Kpnl 695 restriction enzymes, respectively. Each injection solution contained $100 \mathrm{ng} / \mu \mathrm{L}$ linearized 696 DNA, $500 \mathrm{ng} / \mu \mathrm{L}$ carrier DNA, $0.12 \mathrm{M} \mathrm{KCl,} \mathrm{20 \%} \mathrm{glycerol,} \mathrm{0.1 \%} \mathrm{Texas} \mathrm{Red} \mathrm{dextran} \mathrm{in}$ DNAse-free, sterile water. S. purpuratus embryos were cultured for $48 \mathrm{hpf}$ and $L$. variegatus were cultured for $28 \mathrm{hpf}$ before being mounted for live imaging. Embryos were scored to determine the total number of injected embryos (indicated by the presence of Texas Red dextran), the number of embryos showing PMC-specific GFP expression, the number of embryos showing PMC and ectopic GFP expression, and the number of

702 embryos with only ectopic GFP expression.

703

704

705

706

707

708

709

710

711

712

713

714

715

716

717

718

719

720

721

722

723

724

725

726

727

728

NanoString analysis

Direct quantitative measurement of GFP and mCherry RNA transcripts and incorporated DNA was performed using the Nanostring nCounter Elements XT protocol. Briefly, a pair of target-specific oligonucleotide pairs (Probes $A$ and $B$ ) complementary to each target gene and transcript were synthesized by Integrated DNA Technologies (Coralville, IA, USA). Probes $A$ and $B$ also included short tails complementary to NanoString Reporter Tags and Universal Capture Tags, respectively. RNA targets included GFP, mCherry, and several $S$. purpuratus housekeeping genes (foxJ1, hlf, kazL, and rasprp3) that represented a range of transcript abundances and that were expressed at constant levels over the developmental time window of interest. DNA targets included GFP, mCherry, several endogenous, single-copy genes (hypp_1164, hypp_1901, hypp_2956, hypp_592, kirrelL), and one multi-copy gene (pmar1). DNA probes were complementary to the noncoding DNA strand to avoid hybridization to RNA. Probe sequences are available in Supplemental Table S3. For detection, we used the NanoString Elements XT Reporter Tag Set-12 and Universal Capture Tag.

Embryos injected with parental and mutant BACs were harvested at 20, 30, 50 and 65 hpf using the Qiagen AllPrep DNA/DNA micro kit. An additional on-column DNase treatment was included in the RNA recovery process to remove contaminating DNA. Genomic DNA extracted was sonicated using a Bioruptor Pico (Diagenode) for 6 minutes (30 seconds $\mathrm{ON}, 30$ seconds OFF) at $4^{\circ} \mathrm{C}$ to obtain 200 bp fragments (confirmed using an Agilent Bioanalyzer). Sonicated DNA was extracted using ethanol precipitation. GFP or mCherry RNA counts were first normalized to housekeeping transcript counts. DNA counts were normalized to single copy gene counts to obtain number of incorporated DNA per nucleus. To obtain RNA count per incorporated DNA for each sample, normalized RNA counts were divided by normalized incorporated DNA counts (Supplemental Table S2 and S4). 
729 Whole-mount in situ hybridization

730 DNA templates for RNA probe synthesis were amplified with reverse primers that 731 contained T3 promoter (see Table S3). Invitrogen MEGAscript T3 Transcription Kit was 732 then used to amplify digoxigenin-labeled RNA from the DNA templates. Whole-mount in 733 situ hybridization (WMISH) was performed as previously described (Ettensohn et al., 734 2007), with minor modifications. Embryos were collected fixed at the desired stage and 735 fixed 4\% PFA in artificial seawater (ASW) for 1 hour at room temperature. The embryos 736 were then washed twice in ASW and permeabilized and stored in with 100\% methanol. 737 Embryos were then rehydrated and incubated with $1 \mathrm{ng} / \mu \mathrm{L}$ RNA probe overnight at $55^{\circ} \mathrm{C}$. 738 The following day, the embryos were incubated in blocking buffer (1\% BSA and $2 \%$ horse 739 serum in PBST) and then in blocking buffer with 1:2000 a-DIG-AP antibody. Excess 740 antibody was washed away and color reaction for alkaline phosphatase was carried out. 
Amore G, Davidson EH. 2006. cis-Regulatory control of cyclophilin, a member of the ETS-DRI skeletogenic gene battery in the sea urchin embryo. Developmental Biology 293:555-564. doi:10.1016/j.ydbio.2006.02.024

Arnone MI, Andrikou C, Annunziata R. 2016. Echinoderm systems for gene regulatory studies in evolution and development. Current Opinion in Genetics \& Development 39:129-137. doi:10.1016/j.gde.2016.05.027

Arnone MI, Dmochowski IJ, Gache C. 2004. Using Reporter Genes to Study cis-Regulatory ElementsMethods in Cell Biology. Elsevier. pp. 621-652. doi:10.1016/S0091679X(04)74025-X

Baertsch R, Diekhans M, Kent WJ, Haussler D, Brosius J. 2008. Retrocopy contributions to the evolution of the human genome. BMC Genomics 9:466. doi:10.1186/1471-2164-9-466

Buckley KM, Dong P, Cameron RA, Rast JP. 2018. Bacterial artificial chromosomes as recombinant reporter constructs to investigate gene expression and regulation in echinoderms. Briefings in Functional Genomics 17:362-371. doi:10.1093/bfgp/elx031

Calhoun VC, Stathopoulos A, Levine M. 2002. Promoter-proximal tethering elements regulate enhancer-promoter specificity in the Drosophila Antennapedia complex. Proceedings of the National Academy of Sciences 99:9243-9247. doi:10.1073/pnas.142291299

Cameron RA, Oliveri P, Wyllie J, Davidson EH. 2004. cis-Regulatory activity of randomly chosen genomic fragments from the sea urchin. Gene Expression Patterns 4:205-213. doi:10.1016/j.modgep.2003.08.007

Cary GA, Hinman VF. 2017. Echinoderm development and evolution in the post-genomic era. Developmental Biology 427:203-211. doi:10.1016/j.ydbio.2017.02.003

Cordaux R, Batzer MA. 2009. The impact of retrotransposons on human genome evolution. Nat Rev Genet 10:691-703. doi:10.1038/nrg2640

Czarkwiani A, Dylus DV, Oliveri P. 2013. Expression of skeletogenic genes during arm regeneration in the brittle star Amphiura filiformis. Gene Expression Patterns 13:464-472. doi:10.1016/j.gep.2013.09.002

Davidson EH. 1986. Gene activity in early development. Orlando: Academic Press.

Dermody TS, Kirchner E, Guglielmi KM, Stehle T. 2009. Immunoglobulin Superfamily Virus Receptors and the Evolution of Adaptive Immunity. PLoS Pathog 5:e1000481. doi:10.1371/journal.ppat.1000481

Dylus DV, Czarkwiani A, Blowes LM, Elphick MR, Oliveri P. 2018. Developmental transcriptomics of the brittle star Amphiura filiformis reveals gene regulatory network rewiring in echinoderm larval skeleton evolution. Genome Biol 19:26. doi:10.1186/s13059-018-14028

Erkenbrack EM, Thompson JR. 2019. Cell type phylogenetics informs the evolutionary origin of echinoderm larval skeletogenic cell identity. Commun Biol 2:160. doi:10.1038/s42003019-0417-3

Ettensohn CA. 2013. Encoding anatomy: Developmental gene regulatory networks and morphogenesis: Encoding Anatomy. genesis 51:383-409. doi:10.1002/dvg.22380

Ettensohn CA, Dey D. 2017. KirrelL, a member of the Ig-domain superfamily of adhesion proteins, is essential for fusion of primary mesenchyme cells in the sea urchin embryo. Developmental Biology 421 :258-270. doi:10.1016/j.ydbio.2016.11.006 
Ettensohn CA, Illies MR, Oliveri P, De Jong DL. 2003. Alx1, a member of the Cart1/Alx3/Alx4 subfamily of Paired-class homeodomain proteins, is an essential component of the gene network controlling skeletogenic fate specification in the sea urchin embryo. Development 130:2917-2928. doi:10.1242/dev.00511

Ettensohn CA, Kitazawa C, Cheers MS, Leonard JD, Sharma T. 2007. Gene regulatory networks and developmental plasticity in the early sea urchin embryo: alternative deployment of the skeletogenic gene regulatory network. Development 134:3077-3087. doi:10.1242/dev.009092

Farré D, Martínez-Vicente P, Engel P, Angulo A. 2017. Immunoglobulin superfamily members encoded by viruses and their multiple roles in immune evasion. Eur J Immunol 47:780796. doi:10.1002/eji.201746984

Gao F, Davidson EH. 2008. Transfer of a large gene regulatory apparatus to a new developmental address in echinoid evolution. Proceedings of the National Academy of Sciences 105:6091-6096. doi:10.1073/pnas.0801201105

Gao F, Thompson JR, Petsios E, Erkenbrack E, Moats RA, Bottjer DJ, Davidson EH. 2015. Juvenile skeletogenesis in anciently diverged sea urchin clades. Developmental Biology 400:148-158. doi:10.1016/j.ydbio.2015.01.017

Guerrero-Santoro J, Khor JM, Açıkbaş AH, Jaynes JB, Ettensohn CA. 2021. Analysis of the DNAbinding properties of Alx1, an evolutionarily conserved regulator of skeletogenesis in echinoderms. Journal of Biological Chemistry 297:100901. doi:10.1016/j.jbc.2021.100901

Khor JM, Guerrero-Santoro J, Douglas W, Ettensohn CA. 2021. Global patterns of enhancer activity during sea urchin embryogenesis assessed by eRNA profiling. Genome Res gr.275684.121. doi:10.1101/gr.275684.121

Khor JM, Guerrero-Santoro J, Ettensohn CA. 2019. Genome-wide identification of binding sites and gene targets of Alx1, a pivotal regulator of echinoderm skeletogenesis. Development 146:dev180653. doi:10.1242/dev.180653

Killian CE, Croker L, Wilt FH. 2010. SpSM30 gene family expression patterns in embryonic and adult biomineralized tissues of the sea urchin, Strongylocentrotus purpuratus. Gene Expression Patterns 10:135-139. doi:10.1016/j.gep.2010.01.002

Koga H, Morino Y, Wada H. 2014. The echinoderm larval skeleton as a possible model system for experimental evolutionary biology: Evolution of Echinoderm Larval Skeleton. genesis 52:186-192. doi:10.1002/dvg.22758

Kurokawa D, Kitajima T, Mitsunaga-Nakatsubo K, Amemiya S, Shimada H, Akasaka K. 1999. $\mathrm{HpEts}$, an ets-related transcription factor implicated in primary mesenchyme cell differentiation in the sea urchin embryo. Mechanisms of Development 80:41-52. doi:10.1016/S0925-4773(98)00192-0

Lagha M, Bothma JP, Levine M. 2012. Mechanisms of transcriptional precision in animal development. Trends in Genetics 28:409-416. doi:10.1016/j.tig.2012.03.006

Logan CY, Miller JR, Ferkowicz MJ, McClay DR. 1999. Nuclear beta-catenin is required to specify vegetal cell fates in the sea urchin embryo. Development 126:345-357. doi:10.1242/dev.126.2.345

Lyons DC, Kaltenbach SL, McClay DR. 2012. Morphogenesis in sea urchin embryos: linking cellular events to gene regulatory network states: Sea urchin gastrulation. WIREs Dev Biol 1:231-252. doi:10.1002/wdev.18 
Makabe KW, Kirchhamer CV, Britten RJ, Davidson EH. 1995. Cis-regulatory control of the SM50 gene, an early marker of skeletogenic lineage specification in the sea urchin embryo. Development 121:1957-1970.

Mann K, Poustka AJ, Mann M. 2008. The sea urchin (Strongylocentrotus purpuratus) test and spine proteomes. Proteome Sci 6:22. doi:10.1186/1477-5956-6-22

Mann K, Wilt FH, Poustka AJ. 2010. Proteomic analysis of sea urchin (Strongylocentrotus purpuratus) spicule matrix. Proteome Sci 8:33. doi:10.1186/1477-5956-8-33

Mathelier A, Fornes O, Arenillas DJ, Chen C, Denay G, Lee J, Shi W, Shyr C, Tan G, WorsleyHunt R, Zhang AW, Parcy F, Lenhard B, Sandelin A, Wasserman WW. 2016. JASPAR 2016: a major expansion and update of the open-access database of transcription factor binding profiles. Nucleic Acids Res 44:D110-D115. doi:10.1093/nar/gkv1176

Nishimura Y, Sato T, Morita Y, Yamazaki A, Akasaka K, Yamaguchi M. 2004. Structure, regulation, and function of micro1 in the sea urchin Hemicentrotus pulcherrimus. Dev Genes Evol 214:525-536. doi:10.1007/s00427-004-0442-0

Oliveri P, Carrick DM, Davidson EH. 2002. A Regulatory Gene Network That Directs Micromere Specification in the Sea Urchin Embryo. Developmental Biology 246:209-228. doi:10.1006/dbio.2002.0627

Oliveri P, Tu Q, Davidson EH. 2008. Global regulatory logic for specification of an embryonic cell lineage. Proceedings of the National Academy of Sciences 105:5955-5962. doi:10.1073/pnas.0711220105

Paul CRC, Smith AB. 1984. The Early Radiation and Phylogeny of Echinoderms. Biological Reviews 59:443-481. doi:10.1111/j.1469-185X.1984.tb00411.x

Peng CJ, Wikramanayake AH. 2013. Differential Regulation of Disheveled in a Novel Vegetal Cortical Domain in Sea Urchin Eggs and Embryos: Implications for the Localized Activation of Canonical Wnt Signaling. PLOS ONE 8:e80693. doi:10.1371/journal.pone.0080693

Peter IS, Davidson EH. 2015. Genomic control process: development and evolution. London, UK; San Diego, CA, USA: Academic Press is an imprint of Elsevier.

Pisani D, Feuda R, Peterson KJ, Smith AB. 2012. Resolving phylogenetic signal from noise when divergence is rapid: A new look at the old problem of echinoderm class relationships. Molecular Phylogenetics and Evolution 62:27-34. doi:10.1016/j.ympev.2011.08.028

Rafiq K, Shashikant T, McManus CJ, Ettensohn CA. 2014. Genome-wide analysis of the skeletogenic gene regulatory network of sea urchins. Development 141:2542-2542. doi:10.1242/dev.112763

Rebeiz M, Patel NH, Hinman VF. 2015. Unraveling the Tangled Skein: The Evolution of Transcriptional Regulatory Networks in Development. Annu Rev Genom Hum Genet 16:103-131. doi:10.1146/annurev-genom-091212-153423

Rebeiz M, Tsiantis M. 2017. Enhancer evolution and the origins of morphological novelty. Current Opinion in Genetics \& Development 45:115-123. doi:10.1016/j.gde.2017.04.006

Richardson W, Kitajima T, Wilt F, Benson S. 1989. Expression of an embryonic spicule matrix gene in calcified tissues of adult sea urchins. Developmental Biology 132:266-269. doi:10.1016/0012-1606(89)90222-4 
Shashikant T, Khor JM, Ettensohn CA. 2018a. From genome to anatomy: The architecture and evolution of the skeletogenic gene regulatory network of sea urchins and other echinoderms. genesis 56:e23253. doi:10.1002/dvg.23253

Shashikant T, Khor JM, Ettensohn CA. 2018b. Global analysis of primary mesenchyme cell cisregulatory modules by chromatin accessibility profiling. BMC Genomics 19:206. doi:10.1186/s12864-018-4542-z

Smith AF, Posakony JW, Rebeiz M. 2017. Automated tools for comparative sequence analysis of genic regions using the GenePalette application. Developmental Biology 429:158-164. doi:10.1016/j.ydbio.2017.06.033

Smith SJ, Rebeiz M, Davidson L. 2018. From pattern to process: studies at the interface of gene regulatory networks, morphogenesis, and evolution. Current Opinion in Genetics \& Development 51:103-110. doi:10.1016/j.gde.2018.08.004

Sun Z, Ettensohn CA. 2014. Signal-dependent regulation of the sea urchin skeletogenic gene regulatory network. Gene Expression Patterns 16:93-103. doi:10.1016/j.gep.2014.10.002

Tu Q, Cameron RA, Davidson EH. 2014. Quantitative developmental transcriptomes of the sea urchin Strongylocentrotus purpuratus. Developmental Biology 385:160-167. doi:10.1016/j.ydbio.2013.11.019

Walters J, Binkley E, Haygood R, Romano LA. 2008. Evolutionary analysis of the cis-regulatory region of the spicule matrix gene SM50 in strongylocentrotid sea urchins. Developmental Biology 315:567-578. doi:10.1016/j.ydbio.2008.01.007

Weitzel HE, Illies MR, Byrum CA, Xu R, Wikramanayake AH, Ettensohn CA. 2004. Differential stability of $\beta$-catenin along the animal-vegetal axis of the sea urchin embryo mediated by dishevelled. Development 131:2947-2956. doi:10.1242/dev.01152

Wray GA. 2007. The evolutionary significance of cis-regulatory mutations. Nat Rev Genet 8:206216. doi:10.1038/nrg2063 